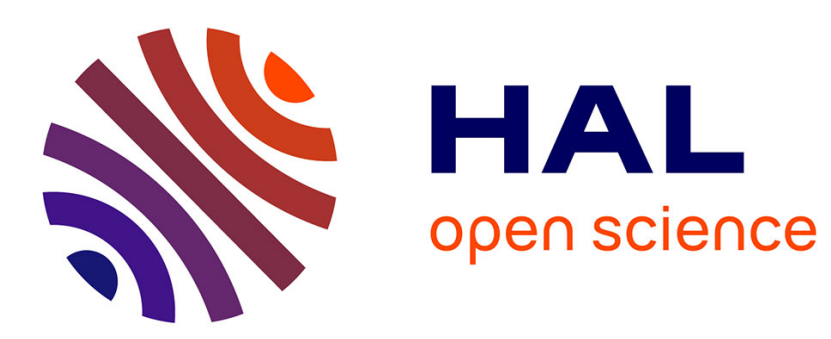

\title{
On Generalized Metric Spaces for the Simply Typed Lambda-Calculus
}

\author{
Paolo Pistone
}

\section{To cite this version:}

Paolo Pistone. On Generalized Metric Spaces for the Simply Typed Lambda-Calculus. LICS 2021 36th Annual ACM/IEEE Symposium on Logic in Computer Science, Jun 2021, Rome, Italy. pp.1-14, 10.1109/LICS52264.2021.9470696 . hal-03346950

\section{HAL Id: hal-03346950 https://hal.science/hal-03346950}

Submitted on 16 Sep 2021

HAL is a multi-disciplinary open access archive for the deposit and dissemination of scientific research documents, whether they are published or not. The documents may come from teaching and research institutions in France or abroad, or from public or private research centers.
L'archive ouverte pluridisciplinaire HAL, est destinée au dépôt et à la diffusion de documents scientifiques de niveau recherche, publiés ou non, émanant des établissements d'enseignement et de recherche français ou étrangers, des laboratoires publics ou privés. 


\title{
On Generalized Metric Spaces for the Simply Typed $\lambda$-Calculus
}

\author{
Paolo Pistone \\ Università di Bologna \\ Email:paolo.pistone2@unibo.it
}

\begin{abstract}
Generalized metrics, arising from Lawvere's view of metric spaces as enriched categories, have been widely applied in denotational semantics as a way to measure to which extent two programs behave in a similar, although non equivalent, way. However, the application of generalized metrics to higher-order languages like the simply typed lambda calculus has so far proved unsatisfactory. In this paper we investigate a new approach to the construction of cartesian closed categories of generalized metric spaces. Our starting point is a quantitative semantics based on a generalization of usual logical relations. Within this setting, we show that several families of generalized metrics provide ways to extend the Euclidean metric to all higher-order types.
\end{abstract}

\section{INTRODUCTION}

In the literature on program semantics much attention has been devoted to program equivalence, and, accordingly, to the study of program transformations which do not produce any observable change of behavior. However, in fields involving numerical or probabilistic forms of computation one often deals with transformations that do alter program behavior, replacing a piece of program with one which is only approximately equivalent. For example, numerical methods (e.g. linear regression, numerical integration) are based on the replacement of computationally expensive operations with more efficient, although less precise, ones. On another scale, statistical learning algorithms compute approximations of a desired function by fitting with a finite sample.

The challenge that accompanies the use of such approximate program transformations [66] is to come up with methods to measure and bound the error they produce. This has motivated much literature on program metrics [6], [65], [8], [31], [9], [25], [19], [26], [35], that is, on semantics in which types are endowed with a notion of distance. This approach has found widespread applications, for example in differential privacy [7], [5], [12] and reinforcement learning [33].

A natural framework for the study of program metrics and their abstract properties is provided by so-called generalized metrics. Since Lawvere's [49] it has been known that some of the basic axioms of standard metric spaces (notably, the reflexivity and transitivity axioms $d(x, x)=0$ and $d(x, z)+d(z, y) \geq d(x, y))$ can be seen, at a higher level of abstraction, as describing the structure of a category enriched over some quantitative algebra. Typically, when this algebra is the usual semi-ring of positive reals (i.e. when " 0 " actually means zero, and "+" actually means plus), one gets the metric spaces everyone is used to. However, one can consider generalized distance functions $d: X \times X \rightarrow Q$, where $Q$ is now a different algebra (typically a quantale or a quantaloid [39]), and the monoidal structure of $Q$ determines the actual meaning of the metric axioms. Well-investigated examples of the generalized approach are given by ultra-metric spaces [65], [31], partial metric spaces [16], [17], [43], [40] and probabilistic metric spaces [60], [38].

Generalized program metrics have been applied in several areas of computer science, e.g. to co-algebraic [11], [45], and concurrent [19] systems, and to algebraic effects [51], [35]. However, the application of program metrics to basic higherorder languages like the simply typed $\lambda$-calculus ST $\lambda \mathrm{C}$ has so far proved unsatisfactory. One can mention both theoretical and practical reasons for this failure. At the abstract level, for instance, there is the well-known fact that standard categories of metric spaces, even generalized, are usually not cartesian closed, and thus only account for linear or sub-exponential variants of ST $\lambda C$ [57], [34], [7]. At a more practical level, there is the observation that even with such restrictions, the distance between two functional programs computed in such models is often not very informative, as it estimates the error of replacing one program by the other one in the worst case, and thus independently of the current context in which these programs are placed.

In this paper we introduce a new class of program metric semantics for ST $\lambda C$ which overcome the aforementioned difficulties. These semantics arise from the study of a class of quantitative models based on what we call quantitative logical relations (in short, QLR).

A QLR is just what remains of a generalized metric space when one discards the reflexivity and transitivity axioms; in other words, it is nothing more than a function $a: X \times X \rightarrow Q$ relating pairs of points $x, y \in X$ with an element $a(x, y)$ of some quantitative algebra $Q$. At the same time, such functions can be seen as a quantitative analog of standard logical relations. The difference is that while with the latter two programs may or may not be related, with QLR two programs are always related to a certain degree.

We believe that models for ST $\lambda \mathrm{C}$ should be as elementary as possible. By the way, the category of sets is itself a denotational model of ST $\lambda$ C. For this reason, we do not, at first, impose any restriction (e.g. continuity, Lipschitz continuity) over the set-theoretic functions between QLR. Importantly, maps of QLR can relate functions measuring distances over different quantitative algebras. For this reason, set-theoretic maps are accompanied by a second map, a sort of derivative, 
relating errors in input with errors in output. In fact, this idea, which extends similar ones from differential logical relations [27], [48] and diameter spaces [36], is the main novelty of our approach with respect to standard metric models (in which one usually considers a fixed quantale), and a key ingredient to obtain models of the full ST $\lambda$ C.

However, recall that our starting point was program metric semantics, and QLR, by their very definition, are not metric spaces. Yet, since generalized metrics are particular cases of QLR, the latter provide an ideal environment to investigate which families of generalized metrics (i.e. which choices of the "O" and the "+") adapt well to the cartesian closed structure.

Our first contribution is to show that several variants of QLR form cartesian closed categories and that some standard results about logical relations (e.g the Fundamental Lemma) have a quantitative analog in the realm of QLR. These results show that QLR-models capture quantitative relational reasoning of higher-order programs in a fully compositional way.

Our second contribution is a characterization of the class of generalized metric spaces that give rise to cartesian closed categories of QLR. These results demonstrate the existence of a variety of compositional metric semantics of ST $\lambda$ C which extend the Euclidean metrics over the reals to all simple types.

Finally, we show that the derivatives found in QLR-models can be compared with those appearing in other quantitative models of ST $\lambda \mathrm{C}$, like those arising from the differential $\lambda$ calculus [29], [14], [15].

Outline: After motivating the introduction of QLR in Section III in Section III we recall the definition of some classes of generalized metric spaces; in Section IV we introduce two cartesian closed categories $\mathbf{Q}$ and $\mathbf{Q}^{r}$ of QLR, and we describe the interpretation of ST $\lambda \mathrm{C}$ in them. In Section V we investigate the generalized metrics which form cartesian closed sub-categories of $\mathbf{Q}$ and $\mathbf{Q}^{r}$. Finally, in Section $\mathrm{V}$ we construct a different cartesian closed category $\mathbf{L L}_{\mathrm{Met}}$ by introducing a "locally Lipschitz" condition for QLR morphisms.

\section{Higher-Order Metric SEMAntics}

\section{A. Program Metrics and Higher-Order Languages}

Program metrics have been widely investigated to capture properties like program similarity and sensitivity. The fundamental idea is usually to associate types $\sigma, \tau$ with metric spaces, and programs $f: \sigma \rightarrow \tau$ with non-expansive, or more generally Lipschitz continuous functions. This means that for all programs $t, u$ of type $\sigma$, the distance between $f(t)$ and $f(u)$ does not exceed that between $t$ and $u$ by more than a fixed factor $L$ (formally, $d(f(t), f(u)) \leq L \cdot d(t, u)$ ).

However, the approach just sketched is not satisfactory for the interpretation of higher-order languages, as those based on ST $\lambda$ C. The main problem is that the category $\operatorname{Met}_{Q}$ of metric spaces over a quantale $Q$ and non-expansive maps [39], which provides the abstract setting for usual program metrics, is not compatible with the usual structure of models of ST $\lambda$ C. More precisely, while the space $\operatorname{Met}_{Q}(X, Y)$ of non-expansive functions can be endowed with a metric (the sup-metric $\left.d_{\text {sup }}(f, g)=\sup \{d(f(x), g(x)) \mid x \in X\}\right)$, this construction does not yield a right-adjoint to the categorical product. For this reason $\operatorname{Met}_{Q}$ is not a cartesian closed category (although $\operatorname{Met}_{Q}$ still admits some interesting cartesian closed sub-categories, see [21], [22]).

This abstract issue is not the only one has to face, though. After all, category theory is usually invoked in program semantics as a way to enforce compositionality, i.e. the property by which the semantics of a composed program is expressed in terms of the semantics of its components. Yet, even if we accept to restrict to higher-order languages compatible with the categorical structure of $\operatorname{Met}_{Q}$ (like e.g. the system Fuzz [57]), the metric $d_{\text {sup }}$ still does not account for the behavior of higher-order programs in a sufficiently compositional, and, in the end, informative way. For example, as observed in [27], consider the two Lipschitz functions $f=\lambda x \cdot \sin (x)$ and $g=\lambda x . x$ : since $f$ and $g$ get arbitrarily far from each other in the worst case (i.e. as $x$ grows to infinity), one can deduce that $d_{\text {sup }}(f, g)$ is infinite. Hence, the distance $d_{\text {sup }}(f, g)$ provides no significant information in any situation in which $f$ is replaced by $g$ as a component of a larger program: for instance, if $\mathrm{C}[\mathrm{]}$ is a context applying a function on values close to 0 , the programs $\mathrm{C}[f]$ by $\mathrm{C}[g]$ will likely turn out close, yet there is no way to predict this fact in terms of $d_{\text {sup }}(f, g)$.

A related issue occurs with contextual notions of distance, as those found e.g. in probabilistc extensions of the $\lambda$-calculus [25]. These metrics extend usual contextual equivalence, by letting the distance $d_{\mathrm{ctx}}(t, u)$ between two objects of type $\sigma$ be the sup of all observable distances $d_{\text {Euc }}(\mathrm{C}[f], \mathrm{C}[g])$, for any context $\mathrm{C}[\mathrm{]}: \sigma \Rightarrow$ Real. In fact, as shown in [26], the nonlinearity of $\mathrm{ST} \lambda \mathrm{C}$ can be used to define contexts that arbitrarily amplify distances, with the consequence that the metric $d_{\text {ctx }}$ trivializes onto plain contextual equivalence.

\section{B. From Program Metrics to Quantitative Logical Relations}

To overcome these issues, in Section IV we introduce quantitative logical relations, a quantitative extension of usual logical relations (generalizing previous approaches [27], [48], [36]) which, on the one hand, applies to higher-order programs without restrictions, and, on the other hand, enables reasoning about behavioral similarity in a fully compositional way.

Semantically, logical relations for a programming language $\mathcal{L}$ can be introduced starting from a denotational model of $\mathcal{L}$ (for simplicity, we consider a simple set-theoretic model, associating each type $\sigma$ with a set $\llbracket \sigma \rrbracket$ and each program $t$ : $\sigma \rightarrow \tau$ with a function $\llbracket t \rrbracket: \llbracket \sigma \rrbracket \rightarrow \llbracket \tau \rrbracket$ ); one then constructs a more refined model whose objects are binary relations $r$ : $\llbracket \sigma \rrbracket \times \llbracket \sigma \rrbracket \rightarrow\{0,1\}$, and whose arrows are those functions from our original model which send related points into related points (in more abstract terms, this construction is an instance of the glueing construction from [42]). The so-called Fundamental Lemma tells then that any program $t: \sigma \rightarrow \tau$ of $\mathcal{L}$ yields a morphism in this model, i.e. preserves relatedness.

While in logical relations relatedness is measured over a fixed algebra (the Boolean algebra $\{0,1\}$ ), in QLR relatedness is measured over a larger class of quantales. Hence, a QLR is 
of the form $a: \llbracket \sigma \rrbracket \times \llbracket \sigma \rrbracket \rightarrow(\sigma)$, where $(\sigma)$ is some quantale associated with $\sigma$. Typically, when $\sigma$ is a functional type, $(\sigma)$ will be some quantale of functions mapping differences in input into differences in output.

To interpret a program $t: \sigma \rightarrow \tau$ we must accompany the function $\llbracket t \rrbracket$ with a second function $(t): \llbracket \sigma \rrbracket \times(\sigma) \rightarrow(\tau)$ mapping differences in $(\sigma)$ around some point of $\llbracket \sigma \rrbracket$ into differences in $(\tau)$. Such functions $(t)$ can be seen as sort of derivatives of the programs of $\mathcal{L}$, and are the key to the compositionality of this semantics: if $\alpha \in(\sigma)$ measures the similarity of two programs $t, u$ and $\mathrm{C}[]: \sigma \rightarrow \tau$ is a context with derivative $(\mathrm{C})$, then by composing $(\mathrm{C})$ with $\llbracket t \rrbracket$ and $\alpha$, we obtain a measure of the similarity between $\mathrm{C}[t]$ and $\mathrm{C}[u]$. Notably, the Fundamental Lemma of logical relations translates in this setting into a result showing that any program $t$ from $\mathcal{L}$ translates into a derivative $(t)$, thus yielding a fully compositional semantics for $\mathcal{L}$.

So for instance, take $\llbracket$ Real $\rrbracket=\mathbb{R}$ and $($ Real $)=\mathbb{R}_{\geq 0}$; if $f, g:$ Real $\rightarrow$ Real are the two programs $\lambda x \cdot \sin (x), \lambda x . x$ seen before and $\mathrm{C}[]=[] 0:($ Real $\rightarrow$ Real $) \rightarrow$ Real is the context that applies a function to 0 , in the setting of quantitative relations we can reason compositionally as follows: first, the difference $d(\llbracket f \rrbracket, \llbracket g \rrbracket)$ will be itself a function, notably one mapping small differences in input around 0 onto small differences in output; secondly, the derivative (C) will be such that that the value $(\mathrm{C})(f, \varphi)$ only depends on how much $\varphi$ grows on small neighborhoods of 0 ; hence, the difference between $\mathrm{C}[f]$ and $\mathrm{C}[g]$, computed by applying $(\mathrm{C})$ to $\llbracket f \rrbracket$ and to $d(\llbracket f \rrbracket, \llbracket g \rrbracket)$, will yield a value close to 0 .

\section{C. ....and back to Generalized Metric Spaces}

While a QLR $a: \llbracket \sigma \rrbracket \times \llbracket \sigma \rrbracket \rightarrow(\sigma)$ needs not be a metric, several classes of generalized metric spaces can be seen as QLR satisfying further properties. One can thus ask which families of generalized metrics can be lifted to all simple types within a given QLR-model.

In Section $\mathrm{V}$ we investigate generalized metrics in categories of QLR with unrestricted morphisms (that is, with no continuity or Lipschitz restriction). We show that, under some mild assumptions, lifting metrics to simple types forces distances to be idempotent (i.e. to satisfy $\alpha=\alpha+\alpha$ ). This implies that the generalized metrics that can be lifted to all simple types are of two kinds: firstly, the ultra-metric and partial ultra-metric spaces, that is, those metrics based on an idempotent quantitative algebra; secondly, those generalized metrics whose distance function can be factored through an idempotent metric. By extending a construction from [36] relating partial metrics with lattice-valued distances, we show that the Euclidean metric, as well as many other standard metrics and partial metrics, belong to this second class.

In Section VI we investigate generalized metrics in categories of QLR where morphisms satisfy suitable generalizations of the Lipschitz and locally Lipschitz continuity conditions. We first show that the first condition does not yield a cartesian closed category, for reasons very similar to those found when considering metrics over a fixed quantale.
We then show that the second yields, instead, a model of $\mathrm{ST} \lambda C$ by restricting to the QLR that satisfy the reflexivity and transitivity axioms, that is, to the generalized metric spaces.

\section{Generalized Metric Spaces}

In this paper we consider several variants of metric spaces. It is thus useful to adopt a general and abstract definition of what we take a (generalized) metric space to be. We exploit the abstract formulation of generalized metric spaces as enriched categories dating back to Lawvere's [49], who first observed that a metric space in the standard sense can be seen as a category enriched in the monoidal poset $([0,+\infty), \geq, 0,+)$ of positve real numbers under reversed ordering and addition.

\section{A. Metrics over an Arbitrary Quantale}

The standard axioms of metric spaces involve an order relation and a monoidal operation (addition) with a neutral element 0 . This structure is characterized by a monoidal poset, that is, a tuple $(M, \geq, 0,+)$ where $(M, \geq)$ is a poset and $(M, 0,+)$ is a monoid such that + is monotone. In practice, one is usually interested in measuring distances in monoidal posets where sups and infs always exist. This leads to consider (commutative and integral) quantales:

Definition III.1. A (commutative) quantale is a commutative monoidal poset $(Q, 0,+, \geq)$ such that $(Q, \geq)$ is a complete lattice satisfying $\alpha+\bigwedge S=\bigwedge\{\alpha+\beta \mid \beta \in S\}$, for all $S \subseteq Q$. A commutative quantale $(Q, 0,+, \leq)$ is integral when $0=\perp$. A commutative quantale $Q$ is a locale when $0=\perp$ and $\alpha=\alpha+\alpha$ holds for all $\alpha \in Q$ (or, equivalently, when $\alpha+\beta=\alpha \vee \beta)$.

Remark III.1. With respect to common presentations of quantales, we adopt here the reversed order (so that $\bigvee s$ and $\bigwedge s$ are inverted), as this is more in accordance with the quantitative intuition.

Example III.1 (The Lawvere quantale). The structure $\left(\mathbb{R}_{\geq 0}^{\infty}, 0,+, \leq\right)$, where $\mathbb{R}_{\geq 0}^{\infty}$ is the set of positive reals plus $\infty$, is a commutative and integral quantale, and usually referred to as the Lawvere quantale [39]. If we replace + with sup, the resulting structure $\left(\mathbb{R}_{\geq 0}^{\infty}, 0, \sup , \leq\right)$ is a locale.

Example III.2. For all commutative monoid $(M, 0,+)$, the structure $(\wp(M),\{0\},+, \subseteq)$, is a commutative quantale, where $A+B=\{x+y \mid x \in A, y \in B\}$.

Example III.3. All products $\Pi_{i \in I} Q_{i}$ of (commutative and integral) quantales, with the point-wise order, are still commutative and integral quantales.

In a quantale $Q$ one can define the following two operations:

$\alpha \circ \beta=\bigwedge\{\delta \mid \beta \otimes \delta \geq \alpha\} \quad \alpha \Leftarrow \beta=\bigwedge\{\delta \mid \beta \vee \delta \geq \alpha\}$

In any quantale $\delta \geq \alpha \circ-\beta$ holds iff $\delta \otimes \beta \geq \alpha$, that is, $\circ$ is right-adjoint to + . A quantale in which $\Leftarrow$ is rightadjoint to $\vee$, i.e. $\delta \geq \alpha \Leftarrow \beta$ holds iff $\delta \vee \beta \geq \alpha$, is called a Heyting quantale [39], [21]. The Lawvere quantale and 
all other quantales obtained from it by product are Heyting. Moreover, all locales are Heyting.

Example III.4. In the Lawvere quantale $x \circ y=$ $\max \{0, x-y\}$ and $x \Leftarrow y$ is 0 if $x \leq y$ and is $x$ otherwise.

Over any quantale $Q$ we can define generalized metric spaces as follows:

Definition III.2. A generalized metric space is a triple $(X, Q, a)$ where $X$ is a set, $Q$ is a commutative quantale, and $a: X \times X \rightarrow Q$ satisfies, for all $x, y, z \in X$ :

$$
\begin{aligned}
0 & \geq a(x, x) \\
a(x, y)+a(y, z) & \geq a(x, z)
\end{aligned}
$$

A generalized metric space is said:

- symmetric if $a(x, y)=a(y, x)$;

- separated if $a(x, y)=0$ implies $x=y$.

Observe that, when $Q$ is integral, from the reflexivity axiom it follows that $a(x, x)=0$ holds for all $x \in X$.

Following usual terminology, we let a pseudo-metric space be a symmetric metric space $(X, Q, a)$, and a standard metric space be a separated pseudo-metric space.

The Euclidean metric is the standard metric space $\left(\mathbb{R}, \mathbb{R}_{\geq 0}^{\infty}, d_{\text {Euc }}\right)$ where $d_{\text {Euc }}(x, y)=|x-y|$.

Example III.5. A standard metric space $(X, Q, a)$ in which $Q$ is a locale is usually called a ultra-metric space. The transitivity axiom reads in this case as $a(x, y) \vee a(y, z) \geq a(x, z)$. For instance, the sequence metric on the set $X^{\mathbb{N}}$ of $X$-sequences $\left(x_{n}\right)_{n \in \mathbb{N}}$ is the ultra-metric space $\left(X^{\mathbb{N}}, \mathbb{R}_{\geq 0}^{\infty}, d_{\text {seq }}\right)$ given by $d_{\text {seq }}\left(x_{n}, y_{n}\right)=2^{-c\left(x_{n}, y_{n}\right)}$, where $c\left(x_{n}, y_{n}\right)$ is the length or the largest common prefix of $x_{n}$ and $y_{n}$.

Example III.6. A standard metric space $(X, \Delta, a)$ in which $\Delta$ is the quantale of distributions, i.e. the left-continuous maps $f: \mathbb{R}_{\geq 0} \rightarrow[0,1]$ with the monoidal operation $(f \oplus g)(r)=$ $\bigwedge_{s+t=r} f(s) \cdot g(s)$, is an example of probabilistic metric space [60], [38]. Observe that the transitivity axiom reads in this case as $a(x, y, r)+a(y, z, s) \geq a(x, y, r+s)$.

\section{B. Partial Metrics Spaces}

In several approaches to program metrics one encounters distance functions which do not satisfy the reflexivity axiom $0 \geq a(x, x)$. A basic example (see [16]) is obtained when the sequence metric $d_{\text {seq }}$ is extended to the set $\widehat{X}=\bigcup_{n}^{\infty} X^{n} \cup$ $X^{\mathbb{N}}$ of finite and infinite $X$-sequences (this kind of spaces are common, for instance, in domain theory): whenever $x_{n}$ is a sequence of length $k$, we have that $d_{\text {seq }}\left(x_{n}, x_{n}\right)=2^{-k}>0$.

The simplest way to define a metric with non-zero selfdistances is simply to drop the reflexivity axiom. This yields the relaxed metrics from [27]. An even more drastic relaxation of the metric axioms is the one considered in [27], where transitivity is also weakened $\mathrm{tc}^{1}$

$$
a(x, y) \leq a(x, z)+a(z, z)+a(z, y)
$$

We will refer to the latter as hyper-relaxed metrics.

A more algebraic approach is to consider distance functions that do satisfy both metric axioms, but relative to a different monoidal structure over $Q$. The partial metric spaces [16], [17], developed to account for domains of objects akin to the set $\widehat{X}$, provide an example of this approach, as shown by the elegant presentation from [40], [64], that we recall below.

For all commutative integral quantale $Q$, let $\mathcal{D}(Q)$ be the category whose objects are all elements of $Q$, and where $\mathcal{D}(Q)(\alpha, \beta)$ is the complete lattice of diagonals from $\alpha$ to $\beta$, i.e. those $\delta \in Q$ satisfying

$$
\alpha+(\delta \circ-\alpha)=\delta=(\delta \circ-\beta)+\beta
$$

The identity morphism $\operatorname{id}_{\alpha}$ is just $\alpha$ (moreover, $\alpha$ is the smallest element of $\mathcal{D}(Q)(\alpha, \alpha)$ ); the composition of two diagonals $\delta \in \mathcal{D}(Q)(\beta, \alpha)$ and $\eta \in \mathcal{D}(Q)(\gamma, \beta)$ is the diagonal

$$
\eta+\beta \gamma:=\eta+(\gamma \circ-\beta)
$$

The category $\mathcal{D}(Q)$ is an example of quantaloid (see [64]).

Example III.7. In the Lawvere quantale, a diagonal from $x$ to $y$ is any real number $z \geq x, y$, and the composition law reads as $x+{ }_{z} y:=x+y-z$.

Remark III.2. When $Q$ is a locale, $\mathcal{D}(Q)(\alpha, \beta)=\{\gamma \mid \alpha \vee$ $\beta \leq \gamma\}$ and the composition law of $\mathcal{D}(Q)$ coincides with that of $Q$, since $\alpha \vee(\beta \Leftarrow \gamma)=\alpha \vee \beta$ holds for all $\gamma \leq \beta$.

Using this fact, the definition of the category of diagonals can be extended to the case in which $Q$ is just a complete lattice (and thus needs not be a locale), by letting $\mathcal{D}(Q)(\alpha, \beta)=\{\gamma \mid \alpha \vee \beta \leq \gamma\}$, with identities $\operatorname{id}_{\alpha}=\alpha$ and composition given by $\vee$. The category $\mathcal{D}(Q)$ is then a quantaloid precisely when $Q$ is a locale.

Partial metric spaces can be defined as metric spaces with respect to the monoidal structure of diagonals:

Definition III.3. $A$ partial metric space is a triple $(X, Q, a)$ where $X$ is a set, $Q$ is a (commutative and integral) quantale and $a: X \times X \rightarrow Q$ satisfies, for all $x, y, z \in X$ :

$$
\begin{aligned}
\operatorname{id}_{a(x, x)} & \geq a(x, x) & \text { (reflexivity) } \\
a(x, y)+_{a(y, y)} a(y, z) & \geq a(x, z) & \text { (transitivity) }
\end{aligned}
$$

A partial metric space is said:

- symmetric if $a(x, y)=a(y, x)$;

- separated if $a(x, y)=a(x, x)=a(y, y)$ implies $x=y$.

A symmetric and separated partial metric over the Lawvere quantale $a: X \times X \rightarrow \mathbb{R}_{\geq 0}^{\infty}$ satisfies the axioms below:

\footnotetext{
${ }^{1}$ Actually, [27] does not define a distance function $d: X \times X \rightarrow Q$ but rather a distance relation $\rho \subseteq X \times Q \times X$ obeying a relaxed transitivity of the form $\rho(x, \alpha, y), \rho(y, \beta, y), \rho(y, \gamma, z) \Rightarrow \rho(x, \alpha+\beta+\gamma, y)$. In fact, this is the same thing as a function $d_{\rho}: X \times X \rightarrow \wp(Q)$ (where $\wp(Q)$ indicates the quantale of subsets of $Q$ from Example III.2) satisfying (1).
} 
PMS1 $a(x, x) \leq a(x, y), a(y, x)$;

PMS2 $a(x, y)=a(y, x)$;

PMS3 if $a(x, x)=a(x, y)=a(y, x)$, then $x=y$;

PMS4 $a(x, y) \leq a(x, z)+a(z, y)-a(z, z)$.

Observe that a (symmetric and separated) metric is the same as a (symmetric and separated) partial metric with $a(x, x)=0$. Moreover, any (symmetric and separated) partial metric $a$ : $X \times X \rightarrow Q$ gives rise to a (symmetric and separated) metric

$$
a^{*}(x, y)=(a(x, y) \circ a(x, x))+(a(x, y) \circ a(y, y))
$$

We let a partial pseudo-metric space be a symmetric metric space, a standard partial metric space be a separated partial pseudo-metric space, and a partial ultra-metric space be a standard partial metric space $(X, Q, a)$ where $Q$ is a locale.

For example, the sequence metric $d_{\text {seq }}$ extended to $\widehat{X}$ yields a partial ultra-metric space. Another standard example of partial metric over the Lawvere quantale is the one defined over the set $\mathcal{I}$ of closed intervals $\{[r, s] \mid r \leq s\}$ by $p\left([r, s],\left[r^{\prime}, s^{\prime}\right]\right)=\max \left\{s, s^{\prime}\right\}-\min \left\{r, r^{\prime}\right\}$.

Remark III.3. The definition of partial ultra-metric spaces can be extended, as we will do in Section $V$ to the case in which $Q$ is just a complete lattice, following Remark III.2. However, one must be careful that all properties that rely on the existence of the right-adjoint $\Leftarrow$ need not hold in this case.

\section{Quantitative Logical Relations}

In this section we introduce two categories $\mathbf{Q}$ and $\mathbf{Q}^{\mathrm{r}}$ of quantitative logical relations.. After describing their cartesian closed structure, we describe the interpretation of ST $\lambda C$ in these categories and we show that some standard results about logical relations scale to QLR in a quantitative sense.

\section{A. Two Categories of $Q L R$}

A quantitative logical relation $(X, Q, a)$ (in short, a $Q L R$ ) is the given of a set $X$, a commutative quantale $Q$ and a function $a: X \times X \rightarrow Q$. A map of quantitative logical relations $(X, Q, a),(Y, R, b)$ is a pair $(f, \varphi)$, where $f: X \rightarrow$ $Y, \varphi: X \times Q \rightarrow R$ and for all $x, y \in X$,

$$
a(x, y) \leq \alpha \Rightarrow b(f(x), f(y)) \leq \varphi(x, \alpha)
$$

QLR and their maps form a category $\mathbf{Q}$ having as identities the pairs $\left(\operatorname{id}_{X}, \lambda x \alpha . \alpha\right)$, and with composition defined by $(g, \psi) \circ$ $(f, \phi)=\left(g \circ f, \psi \circ\left\langle f \circ \pi_{1}, \varphi\right\rangle\right)$.

The category $\mathbf{Q}$ is cartesian closed: given $\operatorname{QLR}(X, Q, a)$ and $(Y, R, b)$,

- their cartesian product is the QLR $(X \times Y, Q \times R, a \times b)$, and the unit is the space $(\{\star\},\{\star\},\langle\star, \star\rangle \mapsto \star)$;

- their exponential is the $\operatorname{QLR}\left(Y^{X}, R^{X \times Q}, d_{a, b}\right)$ where

$$
\begin{array}{r}
d_{a, b}(f, g)(x, \alpha)=\sup \{d(f(x), g(y)), d(f(x), f(y))) \\
\mid a(x, y) \leq \alpha\}
\end{array}
$$

The isomorphism $\mathbf{Q}(Z \times X, Y) \underset{\mathrm{ev}}{\stackrel{\lambda}{\rightleftarrows}} \mathbf{Q}\left(Z, Y^{X}\right)$ defining the cartesian closed structure is given by $\lambda(f, \varphi)=$ $(\lambda(f), \lambda(\varphi))$ and $\operatorname{ev}(f, \varphi)=(\operatorname{ev}(f), \operatorname{ev}(\varphi))$, where

$$
\begin{aligned}
\lambda(f)(z)(x) & =f(\langle z, x\rangle) \\
\lambda(\varphi)(\langle z, \gamma\rangle)(\langle x, \alpha\rangle) & =\varphi(\langle\langle z, x\rangle,\langle\gamma, \alpha\rangle\rangle) \\
\operatorname{ev}(f)(\langle z, x\rangle) & =f(z)(x) \\
\operatorname{ev}(\psi)(\langle\langle z, x\rangle,\langle\gamma, \alpha\rangle\rangle) & =\psi(\langle z, \gamma\rangle)(\langle x, \alpha\rangle)
\end{aligned}
$$

Given QLR $(X, Q, a)$ and $(Y, R, b)$, for all function $f$ : $X \rightarrow Y$ there exists a smallest function $\mathrm{D}(f): X \times Q \rightarrow R$ such that $(f, \mathrm{D}(f)) \in \mathbf{Q}(X, Y)$, defined by

$$
\mathrm{D}(f)(x, \alpha)=\sup \{b(f(x), f(y)) \mid a(x, y) \leq \alpha\}
$$

We call $\mathbf{D}(f)$ the derivative of $f$. Derivatives in $\mathbf{Q}$ satisfy the following properties:

$$
\begin{aligned}
\mathrm{D}\left(\mathrm{id}_{X}\right)(x, \alpha) & =\alpha \\
\mathrm{D}\left(\pi_{i}\right)\left(\left\langle x_{1}, x_{2}\right\rangle,\left\langle\alpha_{1}, \alpha_{2}\right\rangle\right) & =\alpha_{i} \\
\mathrm{D}(\langle f, g\rangle)(x, \alpha) & =\langle\mathrm{D}(f)(x, \alpha), \mathrm{D}(g)(x, \alpha)\rangle \\
\mathrm{D}(g \circ f)(x, \alpha) & \leq \mathrm{D}(g)(f(x), \mathrm{D}(f)(x, \alpha)) \\
\mathrm{D}(\lambda(f))(x, \alpha) & \leq \lambda(\mathrm{D}(f))(x, \alpha) \\
\mathrm{D}(\operatorname{ev}(f))(x, \alpha) & \leq \operatorname{ev}(\mathrm{D}(f))(x, \alpha)
\end{aligned}
$$

Properties (D1)-(D3) recall some of the axioms of Cartesian Differential Categories [14], a well-investigated formalization of abstract derivatives. Property (D4) is a lax version of the chain rule, and properties (D5) and (D6) state that D commutes with the cartesian closed isomorphisms in a lax way.

Remark IV.1. Derivatives in Cartesian Differential Categories are additive in their second variable, i.e. they satisfy $\partial(f)(x, 0)=0$ and $\partial(f)(x, \alpha+\beta)=\partial(f)(x, \alpha)+\partial(x, \beta)$. By contrast, it is not difficult to construct counter-examples to the additivity of $\mathrm{D}(f)$. Let $f, g: \mathbb{R} \rightarrow \mathbb{R}$ be given by

$$
f(x)=\left\{\begin{array}{ll}
x & \text { if }|x| \leq 1 \\
2 x & \text { otherwise }
\end{array} \quad f(x)= \begin{cases}2 x & \text { if }|x| \leq 1 \\
x & \text { otherwise }\end{cases}\right.
$$

Then $6=\mathrm{D}(f)(x, 1+1)>\mathrm{D}(f)(x, 1)+\mathrm{D}(f)(x, 1)=4$ and $6=\mathrm{D}(g)(x, 1+1)<\mathrm{D}(g)(x, 1)+\mathrm{D}(g)(x, 1)=8$.

The distance function on $Y^{X}$ in $\mathbf{Q}$ can be characterized using derivatives as follows: given $\operatorname{QLR}(X, Q, a)$ and $(Y, R, b)$ and functions $f, g \in Y^{X}$, let $\left(2,\{0<\infty\}, d_{\text {disc }}\right)$ be the QLR given by the discrete metric on $2=\{0,1\}$. Let $\mathbf{h}_{f, g}: 2 \times X \rightarrow Y$ be the function given by $\mathbf{h}_{f, g}(0, x)=f(x)$ and $\mathbf{h}_{f, g}(1, x)=g(x)$. A simple calculation yields then:

Lemma IV.1. $d_{a, b}(f, g)(x, \alpha)=\mathrm{D}\left(\mathbf{h}_{f, g}\right)(\langle\langle 0, x\rangle,\langle\infty, \alpha\rangle\rangle)$.

A consequence of Lemma IV.1 is that the self-distance of $f \in Y^{X}$ coincides with its derivative, that is:

$$
d_{a, b}(f, f)=\mathrm{D}(f)
$$

Observe that this property implies that the self-distance of $f$ is (constantly) zero precisely when $f$ is a constant function. 
We now define a category $\mathbf{Q}^{r}$ of reflexive $\mathrm{QLR}$ : $\mathbf{Q}^{r}$ is the full subcategory of $\mathbf{Q}$ made of $\operatorname{QLR}(X, Q, a)$ such that $Q$ is Heyting and $a(x, x)=0$ holds for all $x \in X$.

Remark IV.2. We will make the further assumption that the objects of $\mathbf{Q}^{r}$ satisfy the following property:

$$
\text { if } \alpha \leq \beta \text { then } \beta \leq \beta \Leftarrow \alpha
$$

Notably, the Lawvere quantale and all quantales constructed from it by product satisfy $(\star \star \star)$.

$\mathbf{Q}^{r}$ inherits the cartesian product from $\mathbf{Q}$. Instead, the exponential of $(X, Q, a)$ and $(Y, R, b)$ in $\mathbf{Q}^{\mathrm{r}}$ is the QLR $\left(Y^{X}, R^{X \times Q}, e_{a, b}\right)$, where

$$
e_{a, b}(f, g):=d_{a, b}(f, g) \Leftarrow \mathrm{D}(f)
$$

Observe that $e_{a, b}(f, f)=\mathrm{D}(f) \Leftarrow \mathrm{D}(f)=0$. The isomorphism $\mathbf{Q}^{\mathrm{r}}(Z \times X, Y) \underset{\mathrm{ev}^{r}}{\stackrel{\lambda^{r}}{\rightleftarrows}} \mathbf{Q}^{\mathrm{r}}\left(Z, Y^{X}\right)$ is given by:

$$
\begin{aligned}
\lambda^{\mathrm{r}}(f, \varphi) & =\left(\lambda(f), \lambda(\varphi) \Leftarrow \lambda z \cdot \mathrm{D}\left(f\left(\left\langle z,{ }_{-}\right)\right)\right)\right. \\
\operatorname{ev}^{\mathrm{r}}(f, \varphi) & =\left(\operatorname{ev}(f), \operatorname{ev}(\varphi) \vee \lambda z \cdot \mathrm{D}\left(f(z)\left({ }_{-}\right)\right)\right)
\end{aligned}
$$

Remark IV.3. In the absence of property $(\star \star)$, , reflexive $Q L R$ only form a cartesian lax-closed category [61]. In particular, one has that $\operatorname{ev}^{r}\left(\lambda^{r}(f, \varphi)\right)=\varphi$ and $\lambda^{r}\left(\operatorname{ev}^{r}(f, \psi)\right) \leq \psi$ (in other words, $\beta$-reduction is preserved while $\eta$-reduction decreases the interpretation).

Remark IV.4. In $\mathbf{Q}$ and $\mathbf{Q}^{r}$ we can define "naïve" extensions of the Euclidean metric to all simple types. In particular, this yields the two distance functions $d$ and e on $\mathbb{R}^{\mathbb{R}}$ below:

$$
\begin{aligned}
& d(f, g)(x, \alpha)=\sup \left\{d_{\mathrm{Euc}}(f(x), f(y)), d_{\mathrm{Euc}}(f(x), g(y))\right. \\
& e(f, g)(x, \alpha)= \begin{cases}d(f, g)(x, \alpha) & \text { if } d(f, g)(x, \alpha)>\mathrm{E}(f)(x, \alpha) \\
0 & \text { otherwise }\end{cases}
\end{aligned}
$$

One can also consider categories $\mathbf{Q}^{\mathrm{s}}, \mathbf{Q}^{\text {rs }}$ of symmetric (resp. reflexive and symmetric) QLR. One has the following:

Lemma IV.2. Let $(X, Q, a),(Y, R, b)$ be symmetric $Q L R$. If $R$ is a locale, then their exponential QLR in $\mathbf{Q}$ is still symmetric.

As a consequence, the categories $\mathbf{Q}_{\wedge}^{\mathrm{s}}$ and $\mathbf{Q}_{\wedge}^{\text {sr }}$ of symmetric (resp. reflexive and symmetric) QLR $(X, Q, a)$ where $Q$ is a locale, are cartesian closed subcategories of $\mathbf{Q}, \mathbf{Q}^{\mathrm{r}}$, respectively. We will meet these two categories in the next section.

The locale-valued one is essentially the only case in which symmetric relations inherit the cartesian closed structure of $\mathbf{Q}$ and $\mathbf{Q}^{r}$, as shown be the lemma below.

Lemma IV.3. Let $(X, Q, a),(Y, R, b)$ be symmetric $Q L R$, where $Y$ is injective ([32], [21], see also the Appendix) and $X$ contains two points $v_{0}, v_{1}$ with $a\left(v_{0}, v_{1}\right) \neq 0$. Then, if the exponential of $X$ and $Y$ in $\mathbf{Q}$ is symmetric, then for all $\alpha \in R$ such that $\alpha+\alpha \in \operatorname{Im}(b), \alpha=\alpha+\alpha$.

\section{B. QLR Models}

We now describe the interpretation of the simply typed $\lambda$ calculus inside $\mathbf{Q}$ and $\mathbf{Q}^{r}$. Concretely, this means associating each simple type with a QLR and each typed program with a morphism of QLR. We describe this situation abstractly through the notion of QLR-model, introduced below.

Definition IV.1. Let $\mathbb{C}$ be a cartesian closed category. A QLRmodel (resp. reflexive QLR-model) of $\mathbb{C}$ is a cartesian closed functor $F: \mathbb{C} \rightarrow \mathbf{Q}$ (resp. $F: \mathbb{C} \rightarrow \mathbf{Q}^{r}$ ).

Concretely, a QLR-model consists in the following data:

- for all object $X$ of $\mathbb{C}$, a QLR $\left(\llbracket X \rrbracket,(X), a_{X}\right)$;

- for all morphism $f \in \mathbb{C}(X, Y)$, functions $\llbracket f \rrbracket: \llbracket X \rrbracket \rightarrow$ $\llbracket Y \rrbracket$ and $(f): \llbracket X \rrbracket \times(X) \rightarrow(Y)$ such that $(\llbracket f \rrbracket,(f))$ is a QLR morphism from $\llbracket X \rrbracket$ to $\llbracket Y \rrbracket$,

where the application $f \mapsto(f)$ satisfies equations D1 D6 in a strict sense. Observe that $(f)$ is in general only an approximation of the derivative $\mathrm{D}(\llbracket f \rrbracket)$ (that is, one has $\mathrm{D}(\llbracket f \rrbracket) \leq(f f)$.

We now describe a concrete QLR-model for a simply typed $\lambda$-calculus $\operatorname{ST} \lambda \mathrm{C}\left(\mathcal{F}_{n}\right)$ over a type Real for real numbers. More precisely, simple types are defined by the grammar

$$
\sigma, \tau:=\operatorname{Real}|\sigma \rightarrow \tau| \sigma \times \tau
$$

For all $n>0$, we fix a set $\mathcal{F}_{n}$ of functions from $\mathbb{R}^{n}$ to $\mathbb{R}$. We consider the usual Curry-style simply-typed $\lambda$-calculus, with left and right projection $\pi_{1}$ and $\pi_{2}$, and with pair constructor $\left\langle_{-},\right\rangle_{\text {, }}$, enriched with the following constants: for all $r \in \mathbb{R}$, a constant $\mathrm{r}$ : Real; for all $n>0$ and $f \in \mathcal{F}_{n}$, a constant $\mathrm{f}:$ Real $\rightarrow \cdots \rightarrow$ Real $\rightarrow$ Real.

The usual relation of $\beta$-reduction is enriched with the following rule, extended to all contexts: for all $n>0$, $f \in \mathcal{F}_{n}$, and $r_{1}, \ldots, r_{n} \in \mathbb{R}, \mathrm{fr}_{1} \ldots \mathrm{r}_{n} \longrightarrow_{\beta} \mathrm{s}$, where $s=f\left(r_{1}, \ldots, r_{n}\right)$. By standard arguments [46], this calculus has the properties of subject reduction, confluence and strong normalization.

We let $\Lambda\left(\mathcal{F}_{n}\right)$ be the cartesian closed category whose objects are the simple types and where $\Lambda\left(\mathcal{F}_{n}\right)(\sigma, \tau)$ is the the quotient of the set of closed terms of type $\sigma \rightarrow \tau$ under $\beta \eta$-equivalence, and composition of $[\lambda x . t] \in \Lambda\left(\mathcal{F}_{n}\right)(\sigma, \tau)$ and $[\lambda x . u] \in \Lambda\left(\mathcal{F}_{n}\right)(\tau, \rho)$ is $[\lambda x . u(t x)]$.

We describe a QLR-model of $\operatorname{ST} \lambda \mathrm{C}\left(\mathcal{F}_{n}\right)$ by defining the QLR $\left(\llbracket \sigma \rrbracket,(\sigma), a_{\sigma}\right)$ as shown below (in fact, a similar construction also yields a reflexive QLR-model):

$$
\begin{array}{ll}
\llbracket \text { Real } \rrbracket=\mathbb{R} \quad(\text { Real })=\mathbb{R}_{\geq 0}^{\infty} & a_{\text {Real }}=d_{\text {Euc }} \\
\llbracket \sigma \times \tau \rrbracket=\llbracket \sigma \rrbracket \times \llbracket \tau \rrbracket & a_{\sigma \times \tau}=a_{\sigma} \times a_{\tau} \\
(\sigma \times \tau)=(\sigma) \times(\tau) & \\
\llbracket \sigma \rightarrow \tau \rrbracket=\llbracket \tau \rrbracket^{\llbracket \sigma \rrbracket} & a_{\sigma \rightarrow \tau}=d_{a_{\sigma}, a_{\tau}} \\
(\sigma \rightarrow \tau)=(\tau) \llbracket \sigma \nabla \times(\sigma) &
\end{array}
$$

Given a context $\Gamma=\left\{x_{1}: \sigma_{1}, \ldots, x_{n}: \sigma_{n}\right\}$ and a term $t$ of type $\Gamma \vdash t: \sigma$ (that we take as representative of a class of terms of type $\left.\left(\prod_{i=1}^{n} \sigma_{i}\right) \rightarrow \sigma\right)$, the functions $\llbracket t \rrbracket: \prod_{i=1}^{n} \llbracket \sigma_{i} \rrbracket \rightarrow$ 
$\llbracket \sigma \rrbracket$ and $(t): \prod_{i=1}^{n} \llbracket \sigma_{i} \rrbracket \times \prod_{i=1}^{n}\left(\sigma_{i}\right) \rightarrow(\sigma)$ are defined by a straightforward induction on $t$. We unroll below the definition of $(t)$ in the case of $\mathbf{Q}$ :

$$
\begin{aligned}
& (\mathrm{r})(\vec{x}, \vec{\alpha})=0 \\
& (\mathrm{f})(\vec{x}, \vec{\alpha})=\mathrm{D}(f)(\vec{x}, \vec{\alpha}) \\
& (x)(\vec{x}, \vec{\alpha})=\alpha_{i} \\
& (\langle t, u\rangle\rangle(\vec{x}, \vec{\alpha})=\langle(t)(\vec{x}, \vec{\alpha}),(u)(\vec{x}, \vec{\alpha})\rangle \\
& \left.\left(t \pi_{i}\right)(\vec{x}, \vec{\alpha})=\pi_{i}(\mid t t)(\vec{x}, \vec{\alpha})\right) \\
& (\lambda y . t)(\vec{x}, \vec{\alpha})=\lambda x \alpha \cdot(t)(\vec{x} * x, \vec{\alpha} * \alpha) \\
& (t u)(\vec{x}, \vec{\alpha})=(t)(\vec{x}, \vec{\alpha})(\llbracket u \rrbracket(\vec{x}),(u \downarrow)(\vec{x}, \vec{\alpha}))
\end{aligned}
$$

where $\vec{x} * y$ indicates the concatenation of $\vec{x}$ with $y$.

Theorem IV.4 (Soundness). For all simply typed term $t$ such that $\Gamma \vdash t: \sigma,(\llbracket t \rrbracket,(t)) \in \mathbf{Q}(\llbracket \Gamma \rrbracket, \llbracket \sigma \rrbracket)$. Moreover, if $t \longrightarrow_{\beta} u$, then $\llbracket t \rrbracket=\llbracket u \rrbracket$ and $(t)=(u)$.

The following fact is an immediate consequence of Theorem IV.4 and Eq. 3), and can be seen as a quantitative analog of the Fundamental Lemma of logical relations:

Corollary IV.1 (Fundamental Lemma for QLR). For all term $t$ such that $\vdash t: \sigma, a_{\sigma}(\llbracket t \rrbracket, \llbracket t \rrbracket) \leq(t)$.

Another quite literal consequence of Theorem IV.4 is that program distances are contextual: given a distance between programs $t$ and $u$, for any context $\mathrm{C}\left[\_\right]$we can obtain a distance between $\mathrm{C}[t]$ and $\mathrm{C}[u]$ :

Corollary IV.2 (contextuality of distances). For all terms $t, u$ such that $\vdash t, u: \sigma$ holds and for all context $\mathrm{C}[]: \sigma \vdash \tau$,

$$
a_{\tau}(\llbracket \mathrm{C}[t] \rrbracket, \llbracket \mathrm{C}[u] \rrbracket) \leq(\mathrm{C})\left(\llbracket t \rrbracket, a_{\sigma}(\llbracket t \rrbracket, \llbracket u \rrbracket)\right)
$$

Remark IV.5. Corollaries IV.1 and IV.2 generalize properties established in the setting of differential logical relations (cf. Lemma 15 in [27]).

Remark IV.6. One can define an alternative interpretation of $\mathrm{ST} \lambda \mathrm{C}$ by letting $(t)$ be the "true" derivative $\mathrm{D}(\llbracket t \rrbracket)$. However, while Corollaries IV.1 and IV.2 still hold, the operation $t \mapsto$ $(\llbracket t \rrbracket, \mathrm{D}(\llbracket t \rrbracket))$ only yields a colax functor (since one only has $\mathrm{D}(\llbracket u \rrbracket \circ \llbracket t \rrbracket) \leq \mathrm{D}(\llbracket u \rrbracket)(\llbracket t \rrbracket, \mathrm{D}(\llbracket t \rrbracket)))$.

\section{Metrizability}

In this section we investigate generalized metric as subcategories of $\mathbf{Q}$ and $\mathbf{Q}^{r}$. We first show that the relaxed and hyper-relaxed metrics all form cartesian closed subcategories of $\mathrm{Q}$; we then turn to metrics and partial metrics: we show that, under suitable assumptions, the exponential QLR formed from two metric or partial metric spaces $X$ and $Y$ is a metric or a partial metric space precisely when the metric of $Y$ is idempotent (i.e. distances satisfy $\alpha=\alpha+\alpha$ ).

This result can be used to show that the naïve extension to simple types of ultra-metric and partial ultra-metric spaces yields cartesian closed subcategories of $\mathbf{Q}$ and $\mathbf{Q}^{\mathbf{r}}$; at the same time it shows that the naïve extension of the Euclidean metric (as well as of any non-idempotent metric) in either $\mathbf{Q}$ or
$\mathrm{Q}^{\mathrm{r}}$ is not a generalized metric. Nevertheless, we show that extensions to all simple types can be defined for those metrics and partial metrics (including the Euclidean metric), whose distance function factors as the composition of an idempotent metric and a valuation [54], [59].

\section{A. Relaxed metrics}

It is not difficult to check that whenever $(X, Q, a)$ and $(Y, R, b)$ are two relaxed or hyper-relaxed metrics, so is their exponential $\left(Y^{X}, R^{X \times Q}, d\right)$ in $\mathbf{Q}$. For the relaxed metrics, given $f, g, h \in Y^{X}$, using the triangular law of $Y$ we deduce that for all $x, y \in X$ and $\alpha \geq a(x, y)$,

$$
\begin{aligned}
b(f(x), g(y)) & \leq b(f(x), h(x))+b(h(x), g(y)) \\
& \leq d_{a, b}(f, h)(x, \alpha)+d_{a, b}(h, g)(x, \alpha)
\end{aligned}
$$

and thus in particular that $d_{a, b}(f, g) \leq d_{a, b}(f, h)+d_{a, b}(h, g)$. This argument straightforwardly scales to the hyper-relaxed metrics, yielding:

Proposition V.1. The full subcategories of $\mathbf{Q}$ made of relaxed and hyper-relaxed metrics are cartesian closed.

An immediate consequence is that the QLR $\left(\mathbb{R}^{\mathbb{R}},\left(\mathbb{R}_{>0}^{+\infty}\right)^{\left.X \times \mathbb{R}_{\geq 0}^{+\infty}, d\right)}\right.$ from Remark IV.4 is a relaxed metric. We will show below that we cannot actually say more of this QLR: it is not a partial metric.

\section{B. Ultra-metrics}

For all metric spaces $(X, Q, a)$ and $(Y, R, b)$, whenever $R$ satisfies $\alpha+\beta=\alpha \vee \beta$ (or, equivalently, $\alpha=\alpha+\alpha$ and $0=\perp$ ), it is not difficult to check that the transitivity axiom lifts to the exponential in Q: in fact, for all $f, g, h \in Y^{X}$ and $x, y \in X$ with $a(x, y) \leq \alpha)$ one has

$$
\begin{aligned}
b(f(x), g(y)) & \leq b(f(x), h(x)) \vee b(h(x), g(y)) \\
& \leq d_{a, b}(f, h)(x, \alpha) \vee d_{a, b}(h, g)(x, \alpha)
\end{aligned}
$$

from which we deduce $d_{a, b}(f, g)(x, \alpha) \leq d_{a, b}(f, h)(x, \alpha) \vee$ $d_{a, b}(h, g)(x, \alpha)$. A similar argument can be developed for the distance $e_{a, b}$, leading to:

Proposition V.2. The full subcategories of $\mathbf{Q}_{\wedge}^{\mathrm{sr}}$ and $\mathbf{Q}_{\wedge}^{\mathbf{s}}$ made of ultra-metric spaces and partial ultra-metric spaces are cartesian closed.

When $Q$ is a locale, also the category $\operatorname{Met}_{Q}$ is cartesian closed [62]. These categories have been mostly used to provide intensional models of the simply typed $\lambda$-calculus (e.g. measuring program approximations or the number of computation steps [31]). Instead, in the categories $\mathbf{Q}_{\wedge}^{\text {sr }}$ and $\mathbf{Q}_{\wedge}^{\mathrm{s}}$ we can define more extensional metrics as the one below.

Example V.1. Let $\mathcal{I}(\mathbb{R})$ be the complete lattice of closed intervals $[x, y]$ (where $x, y \in \mathbb{R}$ and $x \leq y$ ), enriched with $\emptyset$ and $\mathbb{R}$. We can define a partial ultra-metric $u: \mathbb{R} \times \mathbb{R} \rightarrow \mathcal{I}(\mathbb{R})$ by letting $u(x, y)=[\min \{x, y\}, \max \{x, y\}]$.

The metric u lifts in $\mathbf{Q}_{\wedge}^{\mathbf{s}}$ to a partial ultra-metric $d_{u, u}$ over real-valued functions where, for all $x \in \mathbb{R}$ and $I \in \mathcal{I}(\mathbb{R})$, 
$d_{u, u}(f, g)(x, I)$ is the smallest interval containing all $f(y)$ and $g(y)$, for $y \in I \vee\{x\}$ (see also [36]).

We now establish a sort of converse to Proposition V.2. under suitable conditions, if the exponential of two metric spaces $X$ and $Y$ satisfies the transitivity axiom, then the distances over $Y$ are idempotent:

Lemma V.3. i. Let $(X, Q, a)$ and $(Y, R, b)$ be two metric spaces, where $X$ has at least two distinct points and $Y$ is injective ([32], [21], see also the Appendix). If the reflexive $Q L R\left(Y^{X}, R^{X \times Q}, e_{a, b}\right)$ is a metric space then for all $\alpha, \beta \in R$, if $\alpha+\beta \in \operatorname{Im}(b)$, then $\alpha+\beta=\alpha \vee \beta$.

ii. Let $(X, Q, a)$ and $(Y, R, b)$ be two partial metric spaces, where $X$ has at least two distinct points and $Y$ is injective. If the $Q L R\left(Y^{X}, R^{X \times Q}, d_{a, b}\right)$ is a partial metric space then for all $\alpha, \beta \in R$, if $\alpha+\beta \in \operatorname{Im}(b)$, then $\alpha+\beta=\alpha \vee \beta$.

To give the reader an idea of the proof of Lemma V.3. we illustrate in Fig. 1 counter-examples to transitivity for the naiive extensions of the Euclidean metric (cf. Remark IV.4).

\section{Decomposing Partial Metrics through Valuations}

Lemma V.3 shows that one cannot hope to lift the Euclidean metric to all simple types inside $\mathbf{Q}$ or $\mathbf{Q}^{r}$. Nevertheless, the Euclidean metric, as well as many other non-idempotent metrics and partial metrics, can be lifted to all simple types inside the categories $\mathbf{Q}_{\wedge}^{\mathrm{s}}$ and $\mathbf{Q}_{\wedge}^{\mathrm{sr}}$. We show this fact using a well-investigated connection between partial metrics and lattice-valued metrics.

A basic intuition comes from the observation that the Euclidean distance can be decomposed as

$$
\mathbb{R} \times \mathbb{R} \stackrel{u}{\longrightarrow} \mathcal{I}(\mathbb{R}) \stackrel{\mu}{\longrightarrow} \mathbb{R}_{\geq 0}^{+}
$$

where $u$ is the partial ultra-metric from Example V.1 and $\mu$ is the Lebesgue measure. This observation can be generalized using the theory of valuations [54], [18], [59].

A join-valuation [59] on a join semi-lattice $L$ is a monotone function $\mathcal{F}: L \rightarrow \mathbb{R}_{\geq 0}^{+\infty}$ which satisfies the condition

$$
\mathcal{F}(a \vee b) \leq \mathcal{F}(a)+\mathcal{F}(b)-\mathcal{F}(a \wedge b)
$$

for all $a, b$ such that $a \wedge b$ exists in $L$. When $L$ is a $\sigma$-algebra, join-valuations on $L$ are thus sort of relaxed measures on $L$.

Any join-valuation $\mathcal{F}: L \rightarrow \mathbb{R}_{>0}^{+\infty}$ induces a join semilattice $L_{\mathcal{F}}$ obtained by quotienting $\bar{L}$ under the equivalence

$$
a \simeq_{\mathcal{F}} b \text { iff }(a \leq b \text { or } b \leq a) \text { and } \mathcal{F}(a)=\mathcal{F}(b)
$$

One obtains then a separated and symmetric partial metric $p_{\mathcal{F}}: L_{\mathcal{F}} \times L_{\mathcal{F}} \rightarrow \mathbb{R}_{>0}^{+\infty}$ by letting $p_{\mathcal{F}}(a, b)=\mathcal{F}(a \vee b)$. The transitivity axiom is checked as follows:

$$
\begin{aligned}
\mathcal{F}(a \vee b) & \leq \mathcal{F}((a \vee c) \vee(c \vee b)) \\
& \leq \mathcal{F}(a \vee c)+\mathcal{F}(c \vee b)-\mathcal{F}((a \vee c) \wedge(c \vee b)) \\
& \leq \mathcal{F}(a \vee c)+\mathcal{F}(c \vee b)-\mathcal{F}(c \vee c)
\end{aligned}
$$

Remark V.1. The connection between partial metrics and valuations has a converse side [59]: any (symmetric and separated) partial metric $p: X \times X \rightarrow \mathbb{R}_{>0}^{+\infty}$ defines an order $\sqsubseteq_{p}$ over $X$ given by $x \sqsubseteq_{p} y$ iff $p(x, y) \leq p(x, x)$. Then, whenever the poset $\left(X, \sqsubseteq_{p}\right)$ is a join semi-lattice, the selfdistance function $X \stackrel{\Delta}{\rightarrow} X \times X \stackrel{p}{\rightarrow} \mathbb{R}_{\geq 0}^{+\infty}$ is a join-valuation.

Extending this observation to arbitrary (commutative and integral) quantales leads to the following:

Definition V.1. $A$ (generalized) valuation space (noted $L \stackrel{\mathcal{F}}{\longrightarrow}$ $Q)$ is the given of a monotone function from a complete lattice $L$ to a quantale $Q$ satisfying

$$
\mathcal{F}(a \vee b) \leq \mathcal{F}(a)+(\mathcal{F}(b) \circ \mathcal{F}(a \wedge b))
$$

for all $a, b \in L$ such that $a \wedge b \neq \perp$.

By arguing as above, any valuation space $L \stackrel{\mathcal{F}}{\longrightarrow} Q$ yields a (symmetric and separated) partial metric $\mathcal{F}: L_{\mathcal{F}} \times L_{\mathcal{F}} \rightarrow Q$. This leads to the following definition:

Definition V.2. A partial metric valuation space is a triple $(X, L \stackrel{\mathcal{F}}{\longrightarrow} Q, a)$, where $L \stackrel{\mathcal{F}}{\longrightarrow} Q$ is a valuation space and $U X=\left(X, L_{\mathcal{F}}, a\right)$ is a (symmetric and separated) partial ultra-metric space.

A map of partial metric valuation spaces $(X, L \stackrel{\mathcal{F}}{\longrightarrow} Q, a)$ and $(Y, M \stackrel{\mathcal{G}}{\longrightarrow} R, b)$ is an arrow $(f, \varphi)$ in $\mathbf{Q}_{\wedge}^{\mathrm{s}}(U X, U Y)$.

Example V.2. The Euclidean metric can be presented as a partial metric valuation space in two ways: either using the Lebesgue measure as shown before, or by considering the valuation space $\mathcal{I}(\mathbb{R})^{-} \stackrel{\text { diam }}{\longrightarrow} \mathbb{R}_{\geq 0}^{+\infty}$ where $\mathcal{I}(\mathbb{R})^{-}$is the joinsemilattice $\mathcal{I}(\mathbb{R})-\{\emptyset\}$ and diam is the diameter function (which is in fact modular over intersecting intervals, see [36]).

Observe that for any map $(f, \varphi)$ of spaces $(X, L \stackrel{\mathcal{F}}{\longrightarrow} Q, a)$ and $(Y, M \stackrel{\mathcal{G}}{\longrightarrow} R, b)$, we have that for all $x, y \in X$ and $\alpha \in L$,

$$
\mathcal{G}(b(f(x), f(y)) \leq \mathcal{G}(\varphi(x, \alpha))
$$

In other words, the composition of derivatives and valuations provides a compositional way to compute distance bounds.

We let $p V$ indicate the category of partial metric valuation spaces. Since the functor $U: \mathbf{p V} \rightarrow \mathbf{Q}_{\wedge}^{\mathrm{s}}$ is by definition full and faithful, $\mathrm{pV}$ inherits the cartesian closed structure from $\mathbf{Q}_{\wedge}^{\mathrm{s}}$. In particular, given partial metric valuation spaces $(X, L \stackrel{\mathcal{F}}{\longrightarrow} Q, a)$ and $(Y, M \stackrel{\mathcal{G}}{\longrightarrow} R, b)$, their product and exponential are as follows:

$$
\begin{gathered}
(X \times Y, L \times M \stackrel{\mathcal{F} \times \mathcal{G}}{\longrightarrow} R \times Q, a \times b) \\
\left(Y^{X},\left(R_{\mathcal{G}}\right)^{X \times L_{\mathcal{F}}} \stackrel{\mathcal{G}_{-}}{\longrightarrow} Q^{X \times L_{\mathcal{F}}}, d_{a, b}\right)
\end{gathered}
$$

Example V.3. The exponential object of the Euclidean metric inside $\mathrm{pV}$ is (essentially) the partial metric $p:\left(\mathbb{R}^{\mathbb{R}} \times \mathbb{R}^{\mathbb{R}}\right) \rightarrow$ $\left(\mathbb{R}_{\geq 0}^{+}\right)^{\mathbb{R} \times \mathcal{I}(\mathbb{R})}$ from [36], and is given by

$$
p(f, g)(x, I)=\operatorname{diam}\{b(f(y), g(z)) \mid y, z \in\{x\} \vee I\}
$$

We can compare this extension with the naïve extension $d$ in Fig. 1, by considering the interval $I=[x-r, x+r]$. One has 


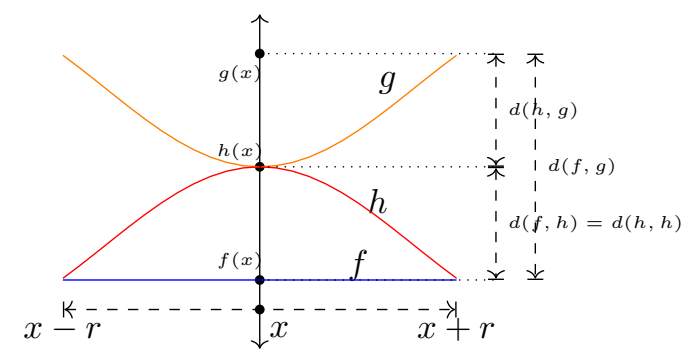

(a) The distance $d$ from Remark IV.4 is not a partial metric. The example above shows that $d(f, g)>d(f, h)+d(h, g)-d(h, h)$ (with all distances computed in $(x, r)$ ). A similar example can be found in [36].

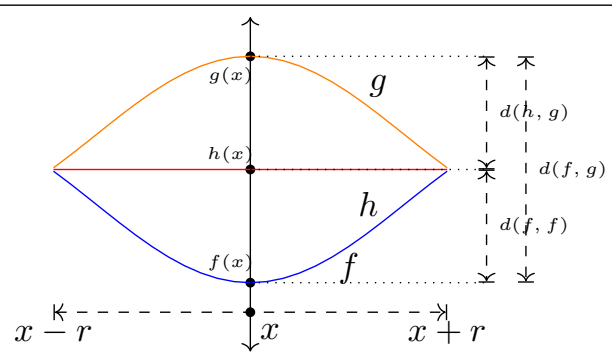

(b) The distance $e$ from Remark IV.4 is not a metric. In the example above (with all values computed in $(x, r)), e(f, h)=0$, since each $h(y)$ is no farther from $f(x)$ than $f(x+r), e(h, g)=d(h, g)$ and $e(f, g)$ is $d(f, f)+d(f, g)$. Hence transitivity fails since $e(f, g)=$ $d(f, h)+d(h, g)>0+d(h, g)=e(f, h)+e(h, g)$.

Fig. 1: The distances $d$ and $e$ from Remark IV.4 do not satisfy the transitivity axioms of metric and partial metric spaces.

$p(f, h)(x, I)=d(f, h)$ but $p(h, g)(x, I)=d(h, h)+d(h, g)$. Hence transitivity holds, since $p(f, g)(x, I)=p(f, h)(x, I)+$ $p(h, g)(x, I)-p(h, h)(x, I)$.

This construction can be adapted to metric spaces. Let a dual join-valuation be a monotone map $L^{\mathrm{op}} \times L \stackrel{\mathcal{D}}{\rightarrow} Q$ (where $L^{\mathrm{op}}$ is the complete lattice with the reversed order) satisfying

$$
\mathcal{D}(a, a)=0 \quad \mathcal{D}(a, b \vee c) \leq \mathcal{D}(a, b)+\mathcal{D}(b \wedge c, c)
$$

One defines the quotient $L_{\mathcal{D}}$ by $a \simeq_{\mathcal{D}} b$ iff $a \leq b$ or $b \leq a$ and $\mathcal{D}(a, a \vee b)=\mathcal{D}(b, b \vee a)=0$. For any dual join valuation $\mathcal{D}$, the function $d_{\mathcal{D}}: L_{\mathcal{D}} \times L_{\mathcal{D}} \rightarrow Q$ given by $d(a, b)=\mathcal{D}(a, a \vee b)+\mathcal{D}(b, b \vee a)$ is a symmetric and separated metric. Moreover, any join-valuation $L \stackrel{\mathcal{F}}{\rightarrow} Q$ yields the dual join valuation $\mathcal{F}^{\prime}(a, b)=\mathcal{F}(b) \circ-\mathcal{F}(a)$.

One can define then a metric valuation space as a triple $\left(X, L^{\mathrm{op}} \times L \stackrel{\mathcal{D}}{\rightarrow} Q, a\right)$, where $L^{\mathrm{op}} \times L \stackrel{\mathcal{D}}{\rightarrow} Q$ is a dual join valuation and $U X=\left(X, L_{\mathcal{D}}, a\right)$ is a symmetric and separated ultra-metric space One obtains then a category $\mathbf{V}$ of metric valuation spaces, with $\mathbf{V}(X, Y)=\mathbf{Q}_{\wedge}^{\text {sr }}(U X, U Y)$.

Theorem V.4. The categories $\mathrm{pV}$ and $\mathrm{V}$ are cartesian closed.

Example V.4. The Euclidean metric lives in $\mathbf{V}$ as it arises from the dual join valuation $\mathcal{D}: \mathcal{I}(\mathbb{R})^{\mathrm{op}} \times \mathcal{I}(\mathbb{R}) \rightarrow \mathbb{R}_{>0}^{\infty}$ given by $\mathcal{D}(I, J)=\operatorname{diam}(J) \circ \operatorname{diam}(I)$. Its extension to $\mathbb{R}^{\mathbb{R}}$ inside $\mathbf{V}$ yields the metric $m(f, g)=2 p(f, g)-p(f, f)-p(g, g)$, where $p$ is the partial metric from Example V.3.

\section{A Generalized LiPschitz CONDITION}

In this section we explore a different class of morphisms between QLR, generalizing the usual Lipschitz condition. Notably, we show that in this setting the QLR satisfying reflexivity and transitivity can be lifted to all simple types.

\section{A. From Lipschitz to Locally Lipschitz functions}

As observed in previous sections, the Lipschitz condition has been widely investigated in program semantics, but is considered problematic when dealing with fully higher-order languages. Does the picture change when we step from models like $\operatorname{Met}_{Q}$ to categories of QLR?

Remark VI.1. For simplicity, from now on we will suppose that QLR are always reflexive and symmetric.

To answer this question we must first find a suitable extension of the Lipschitz condition to this setting. The first step is to introduce a notion of finiteness: since a quantale is a complete lattice, we must avoid that any function $f: X \rightarrow Y$ between QLR admits the trivial Lipschitz constant $T$.

Definition VI.1. Let $Q$ be a commutative and integral quantale. A finiteness filter of $Q$ is a downward set $F \subseteq Q$ such that $a, b \in F$ implies $a+b \in F$.

A finitary QRL is a tuple $(X, Q, F, a)$ such that $(X, Q, a)$ is a $Q L R, F \subseteq Q$ is a finitary filter of $Q$ and $\operatorname{Im}(a) \subseteq F$.

The positve reals $\mathbb{R}_{\geq 0}$ form a finitary filter for $\mathbb{R}_{\geq 0}^{\infty}$. Moreover, if $F \subseteq Q$ and $G \subseteq R$ are finitary filters, then $F \times G \subseteq Q \times R$ is a finitary filter of $Q \times R$, and for all set $X, F^{X} \subseteq Q^{X}$ is a finitary filter of $Q^{X}$.

A basic observation is that a Lipschitz $L$ constant for a function $f: X \rightarrow Y$ yields an additive monoid homomorphism $\varphi: \mathbb{R}_{>0}^{+} \rightarrow \mathbb{R}_{>0}^{+}$given by $\varphi(x)=L \cdot x$, such that $d(f(x), f(y)) \leq \varphi(a(x, y))$. This suggests the following:

Definition VI.2 (generalized Lipschitz maps). Let $(X, Q, F, a),(Y, R, G, b)$ be two finitary $Q L R$. A function $f: X \rightarrow Y$ is a generalized Lipschitz map from $X$ to $Y$ if there exists a monoid homomorphism $\varphi: Q \rightarrow R$ satisfying:

$$
\begin{aligned}
& \forall \alpha \in F \varphi(\alpha) \in G \\
& \text { (finiteness) } \\
& a(x, y) \leq \alpha \Rightarrow b(f(x), f(y)) \leq \varphi(\alpha) \\
& \text { (Lipschitz) }
\end{aligned}
$$

Observe that any Lipschitz function $f: \mathbb{R} \rightarrow \mathbb{R}$ in the usual sense is a generalized Lipschitz map between the finitary and reflexive QLR given by the Euclidean metric.

Finitary QLR and generalized Lipschitz maps form a cartesian category $\mathbf{L}$, with cartesian structure defined as similarly to Q. Moreover, given finitary QLR $(X, Q, F, a)$ and $(Y, R, G, b)$ we can define a finitary $\operatorname{QLR}\left(\mathbf{L}(X, Y), R^{X}, G^{X}, b^{X}\right)$ where 
$b^{X}$ is given by $b^{X}(f, g)(x)=b(f(x), g(x))$ (note that also symmetry and reflexivity are preserved).

Yet, with this definition $\mathbf{L}$ is still not cartesian closed. For instance, consider the function $f(x)(y): \mathbb{R} \rightarrow \mathbb{R}^{\mathbb{R}}$ given by $f(x)(y)=x \cdot y$. As a function of two variables, $f$ is Lipschitz in both $x$ and $y$, with Lipschitz constants $|y|$ and $|x|$; one can use this fact to show then that $f \in \mathbf{L}\left(\mathbb{R}, \mathbb{R}^{\mathbb{R}}\right)$. Now, if $\mathbf{L}$ were cartesian closed, using the canonical isomorphism $\mathbf{L}\left(\mathbb{R}, \mathbb{R}^{\mathbb{R}}\right) \simeq \mathbf{L}(\mathbb{R} \times \mathbb{R}, \mathbb{R})$, we could deduce that also the function $\operatorname{ev}(f)(\langle x, y\rangle)=f(x)(y)$ is Lipschitz. However, there is no way to deduce, from the two piecewise Lipschitz constants $|x|$ and $|y|$ for $x$ and $y$, a uniform Lipschitz constant for both variables. In fact, all we can say is that, for any choice of points $x, y \in \mathbb{R}$, we can deduce a Lipschitz constant $L_{x, y}=|x| \cdot|y|$ for $\mathrm{ev}(f)$, although there is no way to define one in a uniform way.

This observation suggests to replace the Lipschitz condition with the local Lipschitz condition. Recall that a function $f: X \rightarrow Y$ between two metric spaces is locally Lipschitz continuous when for all $x \in X$ there exists a constant $L_{x}$ such that the inequality $d(g(y), g(z)) \leq L_{x} \cdot d(y, z)$ holds in some open neighborhood of $x$.

Remark VI.2. From now on we will suppose that quantales $(Q, \geq)$ are continuous as lattices, and we indicate by $\alpha \ll \beta$ the usual way below relation. It is clear that the Lawvere quantale and all quantales obtained from it by applying products are continuous lattices.

The observation above suggests the following definition:

Definition VI.3. Let $(X, Q, F, a)$ and $(Y, R, G, b)$ be finitary $Q L R$. A function $f: X \rightarrow Y$ is said generalized locally Lipschitz (in short $L L$ ), if there exists a function $\varphi: X \times Q \rightarrow R$ (called $a$ family of LL-constants for $f$ ) such that $\varphi\left(x,,_{-}\right)$is additive in its second variable, and the following hold:

$$
\begin{aligned}
& \forall x \in X, \forall \alpha \in F \varphi(x, \alpha) \in G \\
& \forall x, y, z \in X \exists \delta_{x} \gg 0 a(x, y), a(x, z) \leq \delta_{x}, \\
& a(y, z) \leq \alpha \Rightarrow b(f(y), f(z)) \leq \varphi(x, \alpha)
\end{aligned}
$$

(finiteness)

(local Lipschitz)

Any locally Lipschitz function $f: \mathbb{R} \rightarrow \mathbb{R}$ yields a LL-map between the finitary QLR given by the Euclidean metric.

The finitary QLR with LL maps form a category LL: the identity function $\operatorname{id}_{X}$ has the LL constants $\lambda x \epsilon . \epsilon$. Moreover, the composition of LL functions $f: X \rightarrow Y$ and $g: Y \rightarrow Z$ is LL: if $\varphi$ is a family of LL constants for $f$ and $\psi$ is a family of LL constants for $g$, then the map $(x, \epsilon) \mapsto \psi(f(x), \varphi(x, \epsilon))$ is a family of LL constants for $g \circ f$ (observe that identity and composition of LL constants work precisely as in Q).

One can also consider a more "constructive" category $\mathbf{L L}^{*}$ defined as follows. First, for a $\operatorname{QLR}(X, Q, a)$, let $\simeq_{a}$ be the equivalence relation over $X$ defined by $x \simeq_{a} x^{\prime}$ if $a\left(x, x^{\prime}\right)=$ 0 . We indicate by $X / a$ the quotient of $X$ by $\simeq_{a}$. By definition, the QLR $(X / a, Q, a)$ is separated.

Now, the objects of $\mathbf{L L}^{*}$ are the same as those of $\mathbf{L L}$; instead, the arrows between $(X, Q, F, a)$ and $(Y, R, G, b)$ are pairs $(f, \varphi)$, where $f: X \rightarrow Y$ is LL and stable under $\simeq_{a^{-}}$ classes (that is, $a(x, y)=0$ implies $b(f(x), f(y))=0$ ), and $\varphi$ is a family of LL-constants for $f$ (and is also stable under $\simeq_{a}$-classes, that is $a(x, y)=0$ implies $\left.\varphi(x, \alpha)=\varphi(y, \alpha)\right)$.

There is a forgetful functor $U: \mathbf{L L}^{*} \rightarrow \mathbf{L L}$ given by $U(X, Q, F, a)=(X \mathcal{L} a, Q, F, \underset{f}{U} a)$, where $U a([x],[y])=$ $a(x, y)$, and $U(f, \varphi)=\widetilde{f}$, where $\widetilde{f}\left([x]_{a}\right)=[f(x)]_{b}$.

Given finitary QLR $(X, Q, F, a)$ and $(Y, R, G, b)$ we can define the two finitary QLR

$$
\left(\mathbf{L L}(X, Y), R^{X}, G^{X}, b^{X}\right) \quad\left(\mathbf{L L}^{*}(X, Y), R^{X}, G^{X}, b^{X} \circ \pi_{1}\right)
$$

Observe that if $X$ and $Y$ satisfy transitivity, so do $\mathbf{L L}(X, Y)$ and $\mathbf{L} \mathbf{L}^{*}(X, Y)$. In particular, if $Y$ is a standard metric space, $\mathbf{L L}(X, Y)$ is a standard metric space, while $\mathbf{L L}^{*}(X, Y)$ is a pseudo-metric space.

Moreover, if the QLR $X, Y, Z$ satisfy transitivity, we can define an isomorphism $\mathbf{L L}^{*}(Z \times X, Y) \underset{\mathrm{ev}}{\stackrel{\lambda}{\rightleftarrows}} \mathbf{L} \mathbf{L}^{*}\left(Z, \mathbf{L} \mathbf{L}^{*}(X, Y)\right)$ as follows:

- the map $\lambda(f, \varphi)=\left(\left\langle\lambda(f), \lambda_{0}(\varphi)\right\rangle, \lambda_{1}(\varphi)\right)$ is defined by

$$
\begin{aligned}
\lambda(f)(z)(x) & =f(\langle z, x\rangle) \\
\lambda_{0}(\varphi)(z)(\langle x, \epsilon\rangle) & =\varphi(\langle z, x\rangle,\langle 0, \epsilon\rangle) \\
\lambda_{1}(\varphi)(\langle z, \zeta\rangle)(x) & =\varphi(\langle z, x\rangle,\langle\zeta, 0\rangle)
\end{aligned}
$$

- the map ev $(\langle g, \psi\rangle, \chi)=\langle\operatorname{ev}(g), \operatorname{ev}(\psi, \chi)\rangle$ is defined by

$$
\begin{aligned}
\operatorname{ev}(f)(\langle z, x\rangle) & =f(z)(x) \\
\operatorname{ev}(\psi, \chi)(\langle z, x\rangle,\langle\zeta, \epsilon\rangle) & =\chi(\langle z, \zeta\rangle)(x)+\psi(z)(\langle x, \epsilon\rangle)
\end{aligned}
$$

Observe that reflexivity and transitivity are essential for the isomorphism above to hold. In fact, for all $(f, \varphi) \in \mathbf{L L}^{*}(Z \times$ $X, Y)$, to show the validity of

$$
b(f(z, x), f(z, x)) \leq \lambda_{1}(\varphi)(z, 0)(x)=0
$$

one makes essential use of the fact that $b(f(z, x), f(z, x))=$ 0 holds in $Y$ for all $z \in Z$ and $x \in X$. Conversely given $(\langle g, \psi\rangle, \chi) \in \mathbf{L} \mathbf{L}^{*}\left(Z, \mathbf{L} \mathbf{L}^{*}(X, Y)\right)$, to show the validity of

$$
b\left(f(z, x), f\left(z^{\prime}, x^{\prime}\right)\right) \leq \chi\left(z, a\left(z, z^{\prime}\right)\right)(x)+\psi(z)\left(x, a\left(x, x^{\prime}\right)\right)
$$

one makes essential use of the transitivity of $Y$ to deduce it from $b\left(f(z, x), f\left(z^{\prime}, x\right)\right) \leq \chi\left(z, a\left(z, z^{\prime}\right)\right)(x)$ and $b\left(f\left(z^{\prime}, x\right), f\left(z^{\prime}, x^{\prime}\right)\right) \leq \psi(z)\left(x, a\left(x, x^{\prime}\right)\right)$,

All this leads to the following result:

Proposition VI.1. The full sub-category $\mathbf{L L}_{\mathrm{Met}} \hookrightarrow \mathbf{L L}$ of standard metric spaces is cartesian closed. The full subcategory $\mathbf{L L}_{\mathrm{pMet}} \hookrightarrow \mathbf{L} \mathbf{L}^{*}$ of pseudo-metric spaces is cartesian closed. Moreover, the restriction of $U$ as a functor from $\mathbf{L L}_{\mathrm{Met}}$ to $\mathbf{L L}_{\mathrm{pMet}}$ is a cartesian closed functor.

The reason why the category LL is less constructive than $\mathbf{L L}^{*}$ is that to establish its cartesian closure one has to invoke the axiom of choice (see Appendix).

Example VI.1. In LL the space of locally Lipschitz functions $\mathbf{L L}(\mathbb{R}, \mathbb{R})$ is endowed with the pointwise metric $d_{\text {Point }}(f, g)$ : $\mathbb{R} \rightarrow \mathbb{R}_{\geq 0}$, where $d_{\text {Point }}(f, g)(x)=d_{\text {Euc }}(f(x), g(x))$. 


\section{B. QLR Models}

We define QLR models within $\mathbf{L} \mathbf{L}_{\mathrm{Met}}$ and $\mathbf{L} \mathbf{L}_{\mathrm{pMet}}$ similarly to what we did for $\mathbf{Q}$.

Definition VI.4. Let $\mathbb{C}$ be a cartesian closed category. A QLR model of $\mathbb{C}$ is a diagram of cartesian closed functors

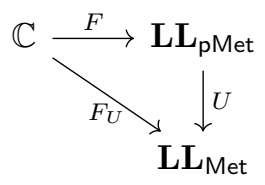

Concretely, a QLR model consists in the following data:

- for all object $X$ of $\mathbb{C}$, a finitary pseudo-metric space $\left(\llbracket X \rrbracket,(X),(X)_{\text {fin }}, a_{X}\right)$

- for all morphism $f \in \mathbb{C}(X, Y)$, a LL-function $\llbracket f \rrbracket$ : $\llbracket X \rrbracket \rightarrow \llbracket Y \rrbracket$ stable on the $a_{X}$-classes, and a family of LL-constants $(f): \llbracket X \rrbracket \times(X) \rightarrow(Y)$ for $\llbracket f \rrbracket$,

where the application $f \mapsto(f)$, which plays the role of the derivative in this setting, satisfies a bunch of properties that we discuss in some more detail below.

We now define a concrete model of the simply typed $\lambda$ calculus over a set of locally Lipschitz functions. For all $n>0$, let us fix a set $\mathcal{L}_{n}$ of locally Lipschitz functions $f: \mathbb{R}^{n} \rightarrow \mathbb{R}$ (in the usual sense), and for each $f \in \mathcal{L}_{n}$, let us fix a function $\operatorname{Lip}(f): \mathbb{R}^{n} \rightarrow[0,+\infty)$ associating each $\vec{x} \in \mathbb{R}^{n}$ with a local Lipschitz constant $\operatorname{Lip}(f)(\vec{x})$ so that when $\vec{y}, \vec{z}$ are in some open neighborhood of $\vec{x}$,

$$
|f(\vec{y})-f(\vec{z})| \leq \operatorname{Lip}(f)(\vec{x}) \cdot d_{\mathrm{Euc}}^{n}(\vec{y}, \vec{z})
$$

where $d_{\text {Euc }}^{n}(\vec{y}, \vec{z})=\sqrt{\sum_{i}\left(y_{i}-z_{i}\right)^{2}}$. For all simple type $\sigma$, the finitary pseudo-metric space $\left(\llbracket \sigma \rrbracket,(\sigma \sigma),(\sigma)_{\text {fin }}, a_{\sigma}\right)$ is defined as follows:

$$
\begin{aligned}
& \llbracket \text { Real } \rrbracket=\mathbb{R} \\
& (\text { Real })=[0, \infty]_{+} \\
& (\text {Real })_{\text {fin }}=\mathbb{R}_{\geq 0}^{\infty} \\
& a_{\text {Real }}=d_{\text {Euc }} \\
& \llbracket \sigma \times \tau \rrbracket=\llbracket \sigma \rrbracket \times \llbracket \tau \rrbracket \\
& (\sigma \times \tau)=(\sigma) \times(\tau) \\
& (\sigma \times \tau)_{\text {fin }}=(\sigma)_{\text {fin }} \times(\tau)_{\text {fin }} \\
& a_{\sigma \times \tau}=a_{\sigma} \times a_{\tau} \\
& \llbracket \sigma \rightarrow \tau \rrbracket=\mathbf{L L}^{*}(\llbracket \sigma \rrbracket, \llbracket \tau \rrbracket) \\
& (\sigma \rightarrow \tau)=(\tau)^{\llbracket \sigma \rrbracket} \\
& \begin{aligned}
(\sigma \rightarrow \tau)_{\text {fin }} & =\left((\mid \tau)_{\text {fin }}\right)^{\llbracket \sigma \rrbracket} \\
a_{\sigma \rightarrow \tau} & =a_{\tau}^{\llbracket \sigma \rrbracket} \circ \pi_{1}
\end{aligned}
\end{aligned}
$$

For all simple type $\sigma, U\left(\llbracket \sigma \rrbracket,(\sigma),(\sigma)_{\text {fin }}, a_{\sigma}\right)$ is then a standard metric space (observe in particular that one has $\left.U a_{\sigma \rightarrow \tau}(f, g)(x)=U a_{\sigma}(f(x), g(x))\right)$.

Given a context $\Gamma=\left\{x_{1}: \sigma_{1}, \ldots, x_{n}: \sigma_{n}\right\}$ and a term $t$ of type $\Gamma \vdash t: \sigma$ (that we take as representative of a class of terms of type $\left(\prod_{i=1}^{n} \sigma_{i}\right) \rightarrow \sigma$ ), the functions $\llbracket t \rrbracket: \prod_{i=1}^{n} \llbracket \sigma_{i} \rrbracket \rightarrow$ $\llbracket \sigma \rrbracket$ and $(t): \prod_{i=1}^{n} \llbracket \sigma_{i} \rrbracket \times \prod_{i=1}^{n}\left(\sigma_{i}\right) \rightarrow(\sigma)$ are defined by a straightforward induction on $t$. We illustrate below only the definition of $(t)$ :

$$
\begin{aligned}
& (\mathrm{r})(\vec{x}, \vec{\alpha})=0 \\
& (\mathrm{f})(\vec{x}, \vec{\alpha})=\operatorname{Lip}(f)(\vec{x}) \cdot\left(\sum \vec{\alpha}\right) \\
& (x)(\vec{x}, \vec{\alpha})=\alpha_{i} \\
& 0\langle t, u\rangle\rangle(\vec{x}, \vec{\alpha})=\langle(t t)(\vec{x}, \vec{\alpha}),(u)(\vec{x}, \vec{\alpha})\rangle \\
& \left(t \pi_{i}\right\rangle(\vec{x}, \vec{\alpha})=\pi_{i}((t t)(\vec{x}, \vec{\alpha})) \\
& (\lambda y . t)(\vec{x}, \vec{\alpha})=\lambda x \cdot(t)(\vec{x} * x, \vec{\alpha} * 0) \\
& (t u)(\vec{x}, \vec{\alpha})=(t)(\vec{x}, \vec{\alpha})(\llbracket u \rrbracket(\vec{x})) \\
& \left.+\llbracket t \rrbracket_{1}(\vec{x}, \vec{\alpha})(\llbracket u \rrbracket(\vec{x}), \mid u \downarrow)(\vec{x}, \vec{\alpha})\right)
\end{aligned}
$$

where we recall that for $t$ of type $\tau \rightarrow \sigma$, $\llbracket t \rrbracket$ is a pair $\left\langle\llbracket t \rrbracket_{0}, \llbracket t \rrbracket_{1}\right\rangle$ with $\llbracket t \rrbracket_{0}(\vec{x}, \vec{\alpha}) \in \llbracket \sigma \rrbracket^{\llbracket \tau \rrbracket}$ and $\llbracket t \rrbracket_{1}(\vec{x}, \vec{\alpha}) \in$ $(\sigma)^{\llbracket \tau \rrbracket \times(\tau)}$.

Theorem VI.2 (Soundness). For all simply typed term $t$ such that $\Gamma \vdash t: \tau,(\llbracket t \rrbracket,(t t)) \in \mathbf{L L}_{\mathrm{pMet}}(\llbracket \Gamma \rrbracket, \llbracket \sigma \rrbracket)$. Moreover, if $t \longrightarrow_{\beta} u$, then $\llbracket t \rrbracket=\llbracket u \rrbracket$ and $(t)=(u)$.

Observe that since the QLR $\left(\llbracket \sigma \rrbracket,(\sigma), a_{\sigma}\right)$ are metric spaces, the Fundamental Lemma reduces in this case to the remark that $a_{\sigma}(\llbracket t \rrbracket, \llbracket t \rrbracket)=0$ holds for all term $t$ of type $\sigma$. Instead, one can prove a "local" version of the contextuality lemma:

Corollary VI.1 (local contextuality of distances). For all terms $t, u$ such that $\vdash t, u: \sigma$ and for all context $\mathrm{C}[]: \sigma \vdash \tau$,

$$
a_{\tau}(\llbracket \mathrm{C}[t] \rrbracket, \llbracket \mathrm{C}[u] \rrbracket) \leq(\mathrm{C})\left(\llbracket t \rrbracket, a_{\sigma}(\llbracket t \rrbracket, \llbracket u \rrbracket)\right)
$$

holds whenever $\llbracket u \rrbracket$ is close enough to $\llbracket t \rrbracket$.

\section{Lipschitz Derivatives and Cartesian Differential Categories}

Due to their different function spaces, the derivatives constructed in $\mathbf{L} \mathbf{L}_{\mathrm{pMet}}$ (i.e. the maps $(t)$ ) behave differently with respect to the derivatives from $\mathbf{Q}$. In particular, the former behave more closely to the derivatives found in Differential $\lambda$-Categories [15] (in short $\mathrm{D} \lambda \mathrm{C}$ ), the categorical models of the differential $\lambda$-calculus [29].

We recall that a $\mathrm{D} \lambda \mathrm{C}$ is a left-additive [14] category $\mathbb{C}$ in which every morphism $f \in \mathbb{C}(X, Y)$ is associated with a morphism $\mathrm{D}(f) \in \mathbb{C}(X \times X, Y)$ satisfying a few axioms: the axioms (D1)-(D7) of Cartesian Differential Categories [14], plus an additional axiom (D-curry) [15] relating derivatives and the function space.

We list below the properties of the application $f \mapsto|f\rangle$ in a QLR model inside $\mathbf{L L}_{\mathrm{pMet}}$. We let $\lambda_{\mathbb{C}}, \mathrm{ev}_{\mathbb{C}}$ indicate the isomorphism $\mathbb{C}(Z \times X, Y) \simeq \mathbb{C}(Z, \mathbb{C}(X, Y))$, and $\mathrm{ev}_{\mathbb{C}}^{*}=$ $\operatorname{ev}_{\mathbb{C}}\left(\operatorname{id}_{\mathbb{C}(X, Y)}\right)$, and similarly ev ${ }^{*}=\operatorname{ev}\left(\operatorname{id}_{\mathbf{L L}_{\mathrm{pMet}}(X, Y)}\right)$ :

(1) $\mid i d l)=\pi_{1},(g \circ f)=(g) \circ\left\langle f \circ \pi_{1},(f f)\right\rangle$;

(2) $(f)(x, 0)=0,(f)(x, \alpha+\beta)=(f)(x, \alpha)+(f)(x, \beta)$;

(3) $\left.\mid \pi_{1}\right)=\pi_{1} \circ \pi_{1},\left(\pi_{2}\right)=\pi_{2} \circ \pi_{1}$;

(4) $0\langle f, g\rangle\rangle=\langle(f f),(\mid g)\rangle$;

(5) $\left(\lambda \lambda_{\mathbb{C}}(f) D=\lambda_{X}(0 f) \circ\left\langle\pi_{1} \times \mathrm{id}_{X}, \pi_{2} \times 0\right\rangle\right)$ (where for $g: Z \times X \rightarrow Y, \lambda_{X}(g)=\lambda x . g\left(\left\langle_{-}, x\right\rangle\right)$ )

(6) $\left(\operatorname{ev}_{\mathbb{C}}^{*} \circ\langle h, g\rangle D=\mathrm{ev}^{*} \circ\left\langle(h), g \circ \pi_{1}\right\rangle+\left(\operatorname{ev}_{\mathbb{C}}(h) D \circ\left\langle\left\langle\pi_{1}, g \circ\right.\right.\right.\right.$ $\left.\left.\pi_{1},\langle 0,(g)\rangle\right\rangle\right)$, (where $h \in \mathbb{C}(Z, \mathbb{C}(X, Y)), g \in \mathbb{C}(Z, X)$ ). 
The properties above literally translate the fact that a QLR model is a cartesian closed functor:

- (1) says that $f \mapsto(f)$ is functorial;

- (2) says that $(f)$ is additive in its second variable;

- (3) and (4) say that the cartesian structure of $\mathbb{C}$ commutes with that of $\mathbf{L L}_{\mathrm{pMet}}$;

- (5) and (6) say that the cartesian closed structure of $\mathbb{C}$ commutes with that of $\mathbf{L L}_{\mathrm{pMet}}$.

(1)-(2)-(3)-(4) coincide with axioms (D2)-(D3)-(D4)-(D5) of Cartesian Differential Categories (in short, CDC). Actually, this is not very surprising, since these axioms describe the fact that the application $f \mapsto \mathrm{D}(f)$ in a CDC $\mathbb{C}$ describe the fact that the application $f \mapsto\langle f, \mathrm{D}(f)\rangle$ yields a cartesian functor (known as the tangent functor, see [24]). Observe that the other axioms of CDCs do not make sense in our setting, because $\mathbf{L L}_{\mathrm{pMet}}$ is not left-additive and there are no "second derivatives" in $\mathbf{L} \mathbf{L}_{\mathrm{pMet}}$.

Finally, property (5) is precisely axiom (D-curry) of $\mathrm{D} \lambda \mathrm{Cs}$, and property (6) can be deduced in any $\mathrm{D} \lambda \mathrm{C}$ from the other axioms (cf. Lemma 4.5, [15]).

\section{RELATED WORKS}

Logical relations [55], [63] are a standard method to establish program equivalence and other behavioral properties of higher-order programs, also related to the concept of relational parametricity [58]. The primary sources of inspiration for the QLR are the differential logical relations [27], [48]. That (non symmetric) differential logical relations are a special cases of QLR can be easily seen as follows: a DLR is a triple $(X, Q, \rho)$, where $Q$ is a quantale and $\rho$ is ternary relation $\rho \subseteq X \times Q \times X$. This is the same as the QLR $\left(X, \wp(Q), d_{\rho}\right)$, where $\wp(Q)$ is the quantale of subsets of $Q$ (see Example [III.2) and $d_{\rho}(x, y)=\{\alpha \mid \rho(x, \alpha, y)\}$. Notably, under this translation, the categorical structure of (non-symmetric) DLR from [27] coincides with the one of the category $\mathbf{Q}$. A precursor of this approach is [66], which develops a System F-based system for approximate program transformations, but without explicitly mentioning any metric structure.

The category $\mathbf{V}$ from Section $\mathbf{V}$ is reminiscent of the diameter spaces from [36], which form a cartesian lax-closed category based on a similar factorization of partial metric spaces. A main difference is that in [36] the factorization is considered as a property of (suitable) partial metric spaces, rather than an additional structure, as we do here.

Several relational logics have been developed to formalize logical relations and, more generally, higher-order relational reasoning [56], [28], [44], [47], [1], including quantitative reasoning [12], [20]. An important question, which transcends the scope of this paper, is whether one can describe a QLR semantics for at least some of these logics, or if a different relational logic has to be developed in order to capture quantitative relational reasoning based on QLR.

The literature on program metrics in denotational semantics is vast. Since [6] metric spaces have been exploited as an alternative framework to standard, domain-theoretic, denotational semantics. Notably, Banach's fixed point theorem plays the role of standard order-theoretic fixpoint theorems in this setting (see [65] and [9]).

More recently, program metrics have been applied in the field of differential privacy [57], [5], [12], by relying on Lipschitz-continuity as a foundation for the notion of program sensitivity. To this line of research belongs also the literature on System Fuzz [57], a sub-exponential PCF-style language designed for differential privacy, which admits an elegant semantics based on metric spaces and metric CPOs [57], [7].

Ultra-metrics are widely applied in program metrics, mostly to describe intensional aspects (e.g. traces, computation steps) [65], [50], [31], also for the $\lambda$-calculus, due to the fact that when $Q$ is a locale, $\operatorname{Met}_{Q}$ is cartesian closed.

Partial metrics were introduced in [16] with the goal of modeling partial objects in program semantics, and independently discovered in sheaf theory as $M$-valued sets [41]. [17] shows that partial metrics and relaxed metrics can be used to characterize the topology of continuous Scott domains with a countable bases. This work was, to our knowledge, the first to acknowledge the correspondence between partial metrics and lattices, which was later developed through the theory of valuations [18], [54], [59]. [43] provides a topological characterization of partial metric spaces. Fuzzy and probabilistic partial metric spaces are well-investigated too [68], [67], [37]. Our description of generalized partial metric spaces was based on the elegant presentation from [40], [64] of such spaces as quantaloid-enriched categories.

Together with standard real-valued metrics, Lawvere's generalized metrics [49] have also played a major role in these research lines. More generally, the abstract investigation of metric spaces as quantale and quantaloid-enriched categories is part of the growing field of monoidal topology [39]. To this approach we can be ascribe the already mentioned description of partial metric spaces from [40], [64], as well as the very general characterization of exponentiable metric spaces and quantaloid-enriched categories in [21], [22].

Quantitative approaches based on generalized metric spaces have been developed for bisimulation metrics [10], [11] and algebraic effects [51], [35]. Generalized metrics based on Heyting quantales have been used to investigate properties of graphs and transition systems (see [45] for a recent survey).

Finally, research on axiomatizations of abstract notions of differentiation has been a very active domain of research in recent years [14], [24], [23], [13], [4], [3], supported by the growth of interest in algorithms based on automatic differentiation. The two notions of derivative discussed in this paper can be compared with two lines of research on abstract differentiation. On the one hand, the derivatives arising from differential logical relations (which essentially coincide with the derivatives from $\mathbf{Q}$ ) have been compared [48] with those found in some recent literature on discrete differentiation (e.g. finite difference operators, Boolean derivatives), and approaches based on the so-called incremental $\lambda$-calculus [2], [3]. On the other hand, the derivatives from Section VI can be compared with the literature on Cartesian Differential Categories, originating in Ehrhard and Regnier's work on 
differential Linear Logic and the differential $\lambda$-calculus [30]. Very recently, Cartesian Difference Categories [3] have been proposed as a framework unifying these two lines of research.

\section{CONCLUSION}

This paper provides just a first exploration of the program metrics semantics that arise from the study of quantitative logical relations, and leaves a considerable number of open questions. We indicate a few natural prosecutions of this work.

While our focus here was only on cartesian closure, it is natural to look for QLR-models with further structure (e.g. coproducts, recursion, monads etc.). For instance, by extending the picture to quantaloid-valued relations [64], one can define a coproduct of QLR with nice properties.

The correspondence between metrics and enriched categories suggests to consider the transitivity axiom as a "vertical" composition law for distances. An interesting question is whether one can define 2-(or even 3-)categories of program distances with a nice compositional structure, in analogy with well-investigated higher-dimensional models in categorical rewriting [52], [53]. At a more formal level, the same observation also suggests to investigate relational logics to formalize the metric reasoning justified by QLR-models, in line with the program logics developed for standard logical relations [56], [28] and for quantitative relational reasoning [44], [47], [1], [12], [20].

\section{REFERENCES}

[1] Alejandro Aguirre, Gilles Barthe, Marco Gaboardi, Deepak Garg, and Pierre-Yves Strub. A relational logic for higher-order programs. Proc. ACM Program. Lang., 1(ICFP), August 2017.

[2] Mario Alvarez-Picallo, Alex Eyers-Taylor, Michael Peyton Jones, and C.-H. Luke Ong. Fixing incremental computation. In Luís Caires, editor, Programming Languages and Systems, pages 525-552, Cham, 2019. Springer International Publishing.

[3] Mario Alvarez-Picallo and Jean-Simon Pacaud Lemay. Cartesian difference categories. In Jean Goubault-Larrecq and Barbara König, editors, Foundations of Software Science and Computation Structures, pages 5776, Cham, 2020. Springer International Publishing.

[4] Mario Alvarez-Picallo and C.-H. Luke Ong. Change actions: Models of generalised differentiation. In Mikołaj Bojańczyk and Alex Simpson, editors, Foundations of Software Science and Computation Structures, pages 45-61, Cham, 2019. Springer International Publishing.

[5] Mário S. Alvim, Miguel E. Andrés, Konstantinos Chatzikokolakis, Pierpaolo Degano, and Catuscia Palamidessi. Differential privacy: On the trade-off between utility and information leakage. In Proceedings of the 8th International Conference on Formal Aspects of Security and Trust, FAST-11, pages 39-54, Berlin, Heidelberg, 2011. SpringerVerlag.

[6] André Arnold and Maurice Nivat. Metric interpretations of infinite trees and semantics of non deterministic recursive programs. Theoretical Computer Science, 11(2):181 - 205, 1980.

[7] Arthur Azevedo de Amorim, Marco Gaboardi, Justin Hsu, Shin-ya Katsumata, and Ikram Cherigui. A semantic account of metric preservation. In Proceedings of the 44th ACM SIGPLAN Symposium on Principles of Programming Languages, POPL 2017, page 545?556, New York, NY, USA, 2017. Association for Computing Machinery.

[8] Marco Azevedo de Amorim, Gaboardi, Arthur, Justin Hsu, and Shin-ya Katsumata. Probabilistic relational reasoning via metrics. In LICS 2019, 2019.

[9] Christel Baier and Mila E. Majster-Cederbaum. Denotational semantics in the cpo and metric approach. Theoretical Computer Science, 135(2):171 - 220, 1994.
[10] Paolo Baldan, Filippo Bonchi, Henning Kerstan, and Barbara König. Behavioral metrics via functor lifting. In 34th International Conference on Foundation of Software Technilogy and Theoretical Computer Science (FSTTCS 2014), volume 29 of Leibniz International Proceedings in Informatics (LIPIcs), pages 403-415, Dagstuhl, Germany, 2014. Schloss Dagstuhl-Leibniz-Zentrum fuer Informatik.

[11] Paolo Baldan, Filippo Bonchi, Henning Kerstan, and Barbara König. Coalgebraic behavioral metrics. Log. Methods Comput. Sci., 14(3), 2018.

[12] Gilles Barthe, Boris Köpf, Federico Olmedo, and Santiago Zanella Béguelin. Probabilistic relational reasoning for differential privacy. In Proceedings of the 39th annual ACM SIGPLAN-SIGACT symposium on Principles of programming languages - POPL '12. ACM Press, 2012.

[13] Richard F. Blute, Robin Cockett, J.S.P. Lemay, and R.A.G. Seely. Differential categories revisited. Applied Categorical Structures, 28:171-235, 2020.

[14] Richard F. Blute, Robin Cockett, and R.A.G. Seely. Cartesian Differential Categories. Theory and Applications of Categories, 22(23):622-672, 2009.

[15] Antonio Bucciarelli, Thomas Ehrhard, and Giulio Manzonetto. Categorical models for simply typed resource calculi. Electronic Notes in Theoretical Computer Science, 265:213 - 230, 2010. Proceedings of the 26th Conference on the Mathematical Foundations of Programming Semantics (MFPS 2010).

[16] Michael Bukatin, Ralph Kopperman, Steve Matthews, and Homeira Pajoohesh. Partial metric spaces. American Mathematical Monthly AMER MATH MON, 116:708-718, 102009.

[17] Michael A. Bukatin and Joshua S. Scott. Towards computing distances between programs via scott domains. In Sergei Adian and Anil Nerode, editors, Logical Foundations of Computer Science, pages 33-43, Berlin, Heidelberg, 1997. Springer Berlin Heidelberg.

[18] Michael A. Bukatin and Svetlana Yu. Shorina. Partial metrics and cocontinuous valuations. In Maurice Nivat, editor, Foundations of Software Science and Computation Structures, pages 125-139, Berlin, Heidelberg, 1998. Springer Berlin Heidelberg.

[19] Konstantinos Chatzikokolakis, Daniel Gebler, Catuscia Palamidessi, and Lili Xu. Generalized bisimulation metrics. In Paolo Baldan and Daniele Gorla, editors, CONCUR 2014 - Concurrency Theory, pages 32-46, Berlin, Heidelberg, 2014. Springer Berlin Heidelberg.

[20] Ezgi Cicek, Weihao Qu, Marco Gaboardi, Gilles Barthe, and Deepak Garg. Bidirectional type checking for relational properties. In PLDI 2019, Proceedings of the 40th ACM SIGPLAN Conference on Programmin Language Design and Implementation, pages 533-547, 2019.

[21] Maria Manuel Clementino and Dirk Hofmann. Exponentiation in vcategories. Topology and its Applications, 153(16):3113 - 3128, 2006. Special Issue: Aspects of Contemporary Topology.

[22] Maria Manuel Clementino, Dirk Hofmann, and Isar Stubbe. Exponentiable functors between quantaloid-enriched categories. Applied Categorical Structures, 17(1):91-101, 2009.

[23] Robin Cockett and J.S.P. Lemay. Cartesian integral categories and contextual integral categories. Electronic Notes in Theoretical Computer Science, 341:45-72, 2018.

[24] Robin Cockett and R.A.G. Seely. The Faà di Bruno construction. Theory and Applications of Categories, 25(15):393-425, 2011.

[25] Raphaëlle Crubillé and Ugo Dal Lago. Metric reasoning about $\lambda$-terms: The affine case. In Proceedings of the 2015 30th Annual ACM/IEEE Symposium on Logic in Computer Science (LICS), LICS '15, page 633?644, USA, 2015. IEEE Computer Society.

[26] Raphaëlle Crubillé and Ugo Dal Lago. Metric reasoning about $\lambda$-terms: The general case. In Hongseok Yang, editor, Programming Languages and Systems, pages 341-367, Berlin, Heidelberg, 2017. Springer Berlin Heidelberg.

[27] Ugo Dal Lago, Francesco Gavazzo, and Akira Yoshimizu. Differential logical relations, part I: the simply-typed case. In 46th International Colloquium on Automata, Languages, and Programming, ICALP 2019, July 9-12, 2019, Patras, Greece, pages 111:1-111:14, 2019.

[28] Derek Dreyer, Amal Ahmed, and Lars Birkedal. Logical step-indexed logical relations. Logical Methods in Computer Science, 7:2-16, 2011.

[29] Thomas Ehrhard and Laurent Regnier. The differential lambda-calculus. Theoretical Computer Science, 309:1-41, 2003.

[30] Thomas Ehrhard and Laurent Regnier. The differential lambda-calculus. Theoretical Computer Science, 309(1):1-41, December 2003.

[31] Martín Hötzen Escardó. A metric model of PCF. In Workshop on Realizability Semantics and Applications, 1999. 
[32] R. Espínola and M. A. Khamsi. Introduction to Hyperconvex Spaces, pages 391-435. Springer Netherlands, Dordrecht, 2001.

[33] Norm Ferns, Prakash Panangaden, and Doina Precup. Bisimulation metrics for continuous Markov decision processes. SIAM Journal of Computing, 60(4):1662-1714, 2011.

[34] Marco Gaboardi, Andreas Haeberlen, Justin Hsu, Arjun Narayan, and Benjamin C. Pierce. Linear dependent types for differential privacy. In Proceedings of the 40th annual ACM SIGPLAN-SIGACT symposium on Principles of programming languages - POPL '13. ACM Press, 2013.

[35] Francesco Gavazzo. Quantitative behavioural reasoning for higher-order effectful programs: Applicative distances. In Proceedings of the 33rd Annual ACM/IEEE Symposium on Logic in Computer Science, LICS '18, page 452?461, New York, NY, USA, 2018. Association for Computing Machinery.

[36] Guillaume Geoffroy and Paolo Pistone. A partial metric semantics of higher-order types and approximate program transformations. In Computer Science Logic 2021 (CSL 2021), volume 183 of LIPICsLeibniz International Proceedings in Informatics, pages 35:1-35:18, 2021.

[37] Jialiang He, Hongliang Lai, and Lili Shen. Towards probabilistic partial metric spaces: Diagonals between distance distributions. Fuzzy Sets and Systems, 370:99 - 119, 2019. Theme: Topology and Metric Spaces.

[38] Dirk Hofmann and C. D. Reis. Probabilistic metric spaces as enriched categories. Fuzzy Sets and Systems, 210:1-21, 2013.

[39] Dirk Hofmann, Gavin J Seal, and W Tholen. Monoidal Topology: a Categorical Approach to Order, Metric and Topology. Cambridge University Press, New York, 2014.

[40] Dirk Hofmann and Isar Stubbe. Topology from enrichment: the curious case of partial metrics. Cahiers de Topologie et Géométrie DIfférentielle Catégorique, LIX, 4:307-353, 2018.

[41] U. Höhle. M-valued Sets and Sheaves over Integral Commutative CLMonoids, pages 33-72. Springer Netherlands, Dordrecht, 1992.

[42] Martin Hyland and Andrea Schalk. Glueing and orthogonality for models of linear logic. Theoretical Computer Science, 294(1-2):183231, 2003.

[43] Gunther Jäger and T. M. G. Ahsanullah. Characterization of quantalevalued metric spaces and quantale-valued partial metric spaces by convergence. Applied General Topology, 19(1):129-144, 2018.

[44] Ralf Jung, David Swasey, Filip Sieczkowski, Kasper Svendsen, Aaron Turon, Lars Birkedal, and Derek Dreyer. Iris: Monoids and invariants as an orthogonal basis for concurrent reasoning. SIGPLAN Not., 50(1):637650, January 2015.

[45] Mustapha Kabil and Maurice Pouzet. Generalized metric spaces. relations with graphs, ordered sets and automata: A survey. To be included in a bool on New Trends in Analysis and Geometry, 2020.

[46] Jean-Louis Krivine. Lambda calculus, types and models. Ellis Horwood, 1993.

[47] Morten Krogh-Jespersen, Kasper Svendsen, and Lars Birkedal. A relational model of types-and-effects in higher-order concurrent separation logic. In Proceedings of the 44th ACM SIGPLAN Symposium on Principles of Programming Languages, POPL 2017, pages 218-231, New York, NY, USA, 2017. Association for Computing Machinery.

[48] Ugo Dal Lago and Francesco Gavazzo. Differential logical relations part II: increments and derivatives. In Gennaro Cordasco, Luisa Gargano, and Adele A. Rescigno, editors, Proceedings of the 21st Italian Conference on Theoretical Computer Science, Ischia, Italy, September 14-16, 2020 , volume 2756 of CEUR Workshop Proceedings, pages 101-114. CEURWS.org, 2020.

[49] F. William Lawvere. Metric spaces, generalized logic, and closed categories. Rendiconti del Seminario Matematico e Fisico di Milano, 43(1):135-166, Dec 1973.

[50] Mila E. Majster-Cederbaum and F. Zetzsche. Towards a foundation for semantics in complete metric spaces. Information and Computation, 90(2):217 - 243, 1991.

[51] Radu Mardare, Prakash Panangaden, and Gordon Plotkin. Quantitative algebraic reasoning. In LICS 2016, 2016.

[52] José Meseguer. Conditional rewriting logig as a unified model of concurrency. Theoretical Computer Science, 96:73-155, 1992.

[53] Hiroyuki Miyoshi. Modelling conditional rewriting logic in structured categories. Electronic Notes in Theoretical Computer Science, 4, 1996.

[54] S.J. O'Neill. Partial metrics, valuations and domain theory. Annals of the New York Academy of Sciences, 806:304-315, 1996.

[55] Gordon Plotkin. $\lambda$-definability and logical relations. Manuscript, 1973.
[56] Gordon Plotkin and Martin Abadi. A logic for parametric polymorphism. In TLCA '93, International Conference on Typed Lambda Calculi and Applications, volume 664 of Lecture Notes in Computer Science, pages 361-375. Springer Berlin Heidelberg, 1993.

[57] Jason Reed and Benjamin C. Pierce. Distance makes the types grow stronger: A calculus for differential privacy. SIGPLAN Not., 45(9):157168, September 2010.

[58] John C. Reynolds. Types, abstraction and parametric polymorphism. In R.E.A. Mason, editor, Information Processing '83, pages 513-523. North-Holland, 1983.

[59] M. P. Schellekens. The correspondence between partial metrics and semivaluations. Theor. Comput. Sci., 315(1):135-149, May 2004.

[60] B. Schweizer and A. Sklar. Probabilistic metric spaces. Probability and Applied Mathematics. North-Holland, 1983.

[61] R.A.G. Seely. Modelling computations: A 2-categorical framework. In Proceedings of the Symposium on Logic in Computer Science (LICS '87), pages 65-71, 1987.

[62] M. B. Smyth. Handbook of Logic in Computer Science, volume 1, chapter Topology, pages 641-761. Clarendon Press, Oxford, 1992.

[63] R. Statman. Logical relations and the typed -calculus. Information and Control, 65(2):85 - 97, 1985

[64] Isar Stubbe. An introduction to quantaloid-enriched categories. Fuzzy Sets and Systems, 256:95 - 116, 2014. Special Issue on Enriched Category Theory and Related Topics (Selected papers from the 33rd Linz Seminar on Fuzzy Set Theory, 2012).

[65] Franck van Breugel. An introduction to metric semantics: operational and denotational models for programming and specification languages. Theoretical Computer Science, 258(1):1 - 98, 2001.

[66] Edwin Westbrook and Swarat Chaudhuri. A semantics for approximate program transformations. https://arxiv.org/abs/1304.5531 2013.

[67] Jiyu Wu and Yueli Yue. Formal balls in fuzzy partial metric spaces. Iranian Journal of Fuzzy Systems, 14(2):155-164, 2017.

[68] Yue Yueli. Separated $\Delta+$-valued equivalences as probabilistic partial metric spaces. Journal of Intelligent and Fuzzy Systems, 28:2715-2724, 082015 . 


\section{APPENDIX}

Proof of Lemma IV.1. We have that

$$
\begin{aligned}
\mathrm{D} & \left(\mathbf{h}_{f, g}\right)(\langle\langle 0, x\rangle,\langle\infty, \alpha\rangle\rangle) \\
\quad & =\sup \left\{b\left(\mathbf{h}_{f, g}(\langle 0, x\rangle), \mathbf{h}_{f, g}(\langle i, y\rangle)\right) \mid d_{\text {disc }}(0, i) \leq \infty, a(x, y) \leq \alpha\right\} \\
\quad & =\sup \{b(f(x), f(y)), b(f(x), g(y)) \mid a(x, y) \leq \alpha\} \\
\quad & =d_{a, b}(f, g)(x, \alpha)
\end{aligned}
$$

Proof of Proposition V.2. Let $(X, Q, a),(Y, R, b)$ be objects of $\mathbf{Q}_{\wedge}^{\text {sr }}$. It suffices to show that the QLR $Y^{X}$ satisfies the triangle inequality. Since $R$ is a locale, $\alpha+\beta=\alpha \vee \beta$ holds for all $\alpha, \beta \in R$. Let $f, g, h \in Y^{X}$. Then we have that $\mathrm{D}(f) \vee\left(d_{a, b}(f, h)+d_{a, b}(h, g)\right)=\left(\mathrm{D}(f) \vee d_{a, b}(f, h)\right) \vee d_{a, b}(h, g)$, so in particular for all $x, y \in X$ and $\alpha \geq a(x, y),\left(\mathrm{D}(f) \vee\left(d_{a, b}(f, g)+d_{a, b}(h, g)\right)\right)(x, \alpha)=\left(\left(\mathrm{D}(f) \vee d_{a, b}(f, g)\right)(x, \alpha)\right) \vee\left(\left(\mathrm{D}(f) \vee d_{a, b}(h, g)\right)(x, \alpha)\right) \geq$ $d_{a, b}(f(x), h(x)) \vee d_{a, b}(h(x), g(y)) \geq d_{a, b}(f(x), g(y))$, from which we deduce that $\left(d_{a, b}(f, g)+d_{a, b}(h, g)\right)(x, \alpha) \Leftarrow$ $\mathrm{D}(f)(x, \alpha) \geq\left(d_{a, b}(f, g)(x, \alpha)\right) \Leftarrow(\mathrm{D}(f)(x, \alpha))=e_{a, b}(f, g)(x, \alpha)$.

$\mathrm{A}$ argument can be developed for $\mathbf{Q}_{\wedge}^{\mathrm{s}}$, using the fact that in a locale $\alpha+\gamma \beta=\alpha \vee \beta$.

Let us recall the notion of injective metric space, that will be essential in our next arguments. A map $f: X \rightarrow X$ between two metric spaces $(X, Q, a),(Y, Q, b)$ over the same quantale is said an extension if for all $x, y \in X, b(f(x), f(y))=a(x, y)$, and non-expansive if for all $x, y \in X, b(f(x), f(y)) \leq a(x, y)$.

A metric space $(X, Q, a)$ is injective when for all non-expansive map $f: Y \rightarrow X$ and extension $e: Y \rightarrow Z$ there exists a non-expansive map $h: Z \rightarrow X$ such that $f=h \circ e$.

Injective metric spaces (also known as hyperconvex metric spaces, see [32]) enjoy several nice properties (see [32]). In particular, they form a cartesian closed subcategory of Met [21], which includes the Euclidean metric. Instead, here we will use such spaces to establish a few somehow negative results.

Proof of Lemma IV.3. Let $\alpha \in R$ and $x_{1}, x_{2} \in Y$ be such that $b\left(x_{1}, x_{2}\right)=\alpha+\alpha$. Let $(Z, R, c)$ be a metric space where $Z=X \cup\left\{u_{0}, u_{3}\right\}$ and $c$ is defined so that $c\left(u_{0}, u_{0}\right)=c\left(u_{3}, u_{3}\right)=0$ and the following hold:

$$
\begin{aligned}
& c\left(u_{0}, u_{1}\right), c\left(u_{0}, u_{2}\right), c\left(u_{0}, u_{3}\right)=\alpha \\
& c\left(u_{1}, u_{2}\right), c\left(u_{2}, u_{3}\right), c\left(u_{3}, u_{1}\right)=\alpha+\alpha
\end{aligned}
$$

Since $Y$ is injective, there exists a non-expansive map $f: Y \rightarrow X$ such that $f \circ \iota=\operatorname{id}_{X}$, where $\iota$ is the injection $\iota: X \rightarrow Z$ (which is obviously an expansion). Hence there exist points $x_{0}, x_{3} \in X$ such that $b\left(x_{0}, x_{1}\right), b\left(x_{0}, x_{2}\right), b\left(x_{0}, x_{3}\right)=\alpha$ and $b\left(x_{1}, x_{2}\right), b\left(x_{2}, x_{3}\right), b\left(x_{3}, x_{1}\right) \leq \alpha+\alpha$.

Let $f, g \in Y^{X}$ be defined by

$$
f(w)=\left\{\begin{array}{ll}
x_{1} & \text { if } w=v_{0} \\
x_{2} & \text { otherwise }
\end{array} \quad g(w)= \begin{cases}x_{3} & \text { if } w=v_{0} \\
x_{4} & \text { otherwise }\end{cases}\right.
$$

where $v_{0}, v_{1}$ are two distinct points of $X$ such that $a\left(v_{0}, v_{1}\right) \neq 0$. If $d_{a, b}(f, g)=d_{a, b}(g, f)$, we deduce that

$$
\begin{aligned}
\alpha & \geq \sup \left\{b\left(f\left(v_{0}\right), f(w)\right), b\left(f\left(v_{0}\right), g(w)\right) \mid a\left(v_{0}, w\right) \leq a\left(v_{0}, v_{1}\right)\right\} \\
& =d_{a, b}(f, g)\left(v_{0}, a\left(v_{0}, v_{1}\right)\right) \\
& =d_{a, b}(g, f)\left(v_{0}, a\left(v_{0}, v_{1}\right)\right) \\
& =\sup \left\{b\left(g\left(v_{0}\right), g(w)\right), b\left(g\left(v_{0}\right), f(w)\right) \mid a\left(v_{0}, w\right) \leq a\left(v_{0}, v_{1}\right)\right\} \\
& =\alpha+\alpha
\end{aligned}
$$

Proof of Lemma IV.2. If $R$ is a locale, then we have that for all $x, y \in X, \alpha \in Q$ with $a(x, y) \leq \alpha, b(g(x), f(y)) \leq$ $b(g(x), f(x)) \vee b(f(x), f(y))=b(f(x), f(y)) \vee b(f(x), g(x)) \leq d_{a, b}(f, g)(x, a(x, y))$ and $b(g(x), g(y)) \leq b(g(x), f(x)) \vee$ $b(f(x), g(y))=b(f(x), g(x)) \vee b(f(x), g(y)) \leq d_{a, b}(f, g)(x, a(x, y))$, since $b$ is symmetric. From this we deduce that $d_{a, b}(g, f)(x, \alpha)=\sup \{b(g(x), g(y)), b(g(x), f(y)) \mid a(x, y) \leq \alpha\} \leq d_{a, b}(f, g)(x, \alpha)$ and conversely.

Proof of Lemma V.3 i.. Let $\alpha, \beta \in R$ and $u_{0}, u_{2} \in Y$ be such that $b\left(u_{0}, u_{2}\right)=\alpha+\beta$. Let $Y^{\prime}=Y \cup\left\{v_{1}\right\}$ and $b^{\prime}$ be as $b$ on $Y$ and satisfying $b\left(u_{0}, v_{1}\right)=\alpha, b\left(v_{1}, u_{2}\right)=\beta$. The injection $\iota: Y \rightarrow Y^{\prime}$ is an expansion, hence, since $Y$ is injective, there 
exists a non-expansive function $f: Y^{\prime} \rightarrow Y$ such that $f \circ \iota=\operatorname{id}_{Y}$. This implies in particular that, by letting $u_{1}:=f\left(v_{1}\right)$, $b\left(u_{0}, u_{1}\right) \leq \alpha, b\left(u_{1}, u_{2}\right) \leq \beta$.

Let now $x_{0}, x_{1}$ be two distinct points in $X$ and let $f, g, h: X \rightarrow Y$ be the following functions: $f(x)$ is constantly $u_{0}$ except for $f\left(x_{1}\right)=u_{1} ; g(x)$ is constantly $u_{2}$ and $h(x)$ is constantly $u_{1}$. We have then that $\mathrm{D}(f)\left(x, a\left(x_{0}, x_{1}\right)\right) \leq \alpha, \mathrm{D}(g)=\mathrm{D}(h)=0$. Moreover, for all $x^{\prime} \in X$ with $a\left(x_{0}, x^{\prime}\right) \leq a\left(x_{0}, x_{1}\right), d\left(f\left(x_{0}\right), f\left(x^{\prime}\right)\right), d\left(f\left(x_{0}\right), h\left(x^{\prime}\right)\right) \leq b\left(u_{0}, u_{1}\right) \leq \mathrm{D}(f)\left(x, a\left(x_{0}, x_{1}\right)\right)=$ $\mathrm{D}(f)\left(x_{0}, a\left(x_{0}, x_{1}\right)\right) \vee 0$, that is $d_{a, b}(f, h)\left(x_{0}, a\left(x_{0}, x_{1}\right)\right) \leq \mathrm{D}(f)\left(x, a\left(x_{0}, x_{1}\right)\right)$, and thus $e_{a, b}(f, h)\left(x, a\left(x_{0}, x_{1}\right)\right)=d_{a, b}(f, h) \Leftarrow$ $\mathrm{D}(f))\left(x_{0}, a\left(x_{0}, x_{1}\right)\right) \leq 0$

Then, since by hypothesis $e_{a, b}$ is a metric, we deduce that

$$
\begin{aligned}
\alpha+\beta & =b\left(u_{0}, u_{2}\right)=b\left(f\left(x_{0}\right), g\left(x_{1}\right)\right) \\
& \leq d_{a, b}(f, g)\left(x_{0}, a\left(x_{0}, x_{1}\right)\right) \\
& \leq\left(\mathrm{D}(f) \vee e_{a, b}(f, g)\right)\left(x_{0}, a\left(x_{0}, x_{1}\right)\right) \\
& \leq\left(\mathrm{D}(f) \vee\left(e_{a, b}(f, h)+e_{a, b}(h, g)\right)\right)\left(x_{0}, a\left(x_{0}, x_{1}\right)\right) \\
& \leq \alpha \vee(0+\beta)=\alpha \vee \beta
\end{aligned}
$$

Proof of Lemma V.3 ii.. As in the proof of point i. let $\alpha, \beta \in R$ and $u_{0}, u_{1}, u_{2} \in Y$ be such that $b\left(u_{0}, u_{1}\right) \leq \alpha, b\left(u_{1}, u_{2}\right) \leq \beta$ and $b\left(u_{0}, u_{2}\right)=\alpha+\beta$. We can suppose w.l.o.g. that $b$ is symmetric.

Let now $x_{0}, x_{1}$ be two distinct points in $X$ and let $f, g, h: X \rightarrow Y$ be the following functions: $f(x)$ is constantly $u_{0}, h(x)$ is constantly $u_{1}$ except for $h\left(x_{1}\right)=u_{0}$ and $g(x)$ is constantly $u_{1}$ except for $g\left(x_{1}\right)=u_{2}$. Then we have that $d_{a, b}(f, g)\left(x_{0}, a\left(x_{0}, x_{1}\right)\right)=b\left(u_{0}, u_{2}\right)=\alpha+\beta, d_{a, b}(f, h)\left(x_{0}, a\left(x_{0}, x_{1}\right)\right)=d_{a, b}(h, h)\left(x_{0}, a\left(x_{0}, x_{1}\right)\right)=b\left(u_{0}, u_{1}\right) \leq \alpha$ and $d_{a, b}(h, g)\left(x_{0}, a\left(x_{0}, x_{1}\right)\right)=b\left(u_{0}, u_{1}\right) \vee b\left(u_{1}, u_{2}\right) \leq \alpha \vee \beta$.

Then, since by hypothesis $d_{a, b}$ is a partial metric, we deduce that

$$
\begin{aligned}
\alpha+\beta & =b\left(u_{0}, u_{2}\right)=b\left(f\left(x_{0}\right), g\left(x_{1}\right)\right) \\
& \leq d_{a, b}(f, g)\left(x_{0}, a\left(x_{0}, x_{1}\right)\right) \\
& \leq\left(\left(d_{a, b}(f, h) \circ-d_{a, b}(h, h)\right)+d_{a, b}(h, g)\right)\left(x_{0}, a\left(x_{0}, x_{1}\right)\right) \\
& =\left(\left(d_{a, b}(h, h) \circ-d_{a, b}(h, h)\right)+d_{a, b}(h, g)\right)\left(x_{0}, a\left(x_{0}, x_{1}\right)\right) \\
& =d_{a, b}(h, g)\left(x_{0}, a\left(x_{0}, x_{1}\right)\right) \leq \alpha \vee \beta
\end{aligned}
$$

Proof of Proposition VI.1. We first check the cartesian closure of $\mathbf{L L}_{\mathrm{pMet}}$.

$\Leftrightarrow)$ the map $\lambda(f, \varphi)=\left(\left\langle\lambda(f), \lambda_{0}(\varphi)\right\rangle, \lambda_{1}(\varphi)\right)$ is defined by

$$
\begin{aligned}
\lambda(f)(z)(x) & =f(\langle z, x\rangle) \\
\lambda_{0}(\varphi)(z)(\langle x, \epsilon\rangle) & =\varphi(\langle z, x\rangle,\langle 0, \epsilon\rangle) \\
\lambda_{1}(\varphi)(\langle z, \zeta\rangle)(x) & =\varphi(\langle z, x\rangle,\langle\zeta, 0\rangle)
\end{aligned}
$$

For all $z \in Z$, then map $\lambda_{0}(\varphi)(z)\left(_{-},{ }_{-}\right)$is additive in its second variable; moreover, for all $z \in Z$ and $x \in X$ there is $\left\langle\zeta_{z}, \epsilon_{x}\right\rangle \gg 0$ (which implies $\zeta_{z} \gg 0$ and $\epsilon_{x} \gg 0$ ) such that, whenever $c\left(z, z^{\prime}\right), c\left(z, z^{\prime \prime}\right) \leq \zeta_{z}$ and $a\left(x, x^{\prime}\right), a\left(x, x^{\prime \prime}\right) \leq \epsilon_{x}$, $\lambda_{0}(\varphi)(z)\left(\left\langle x, a\left(x^{\prime}, x^{\prime \prime}\right)\right\rangle\right) \geq b\left(\lambda(f)(z)\left(x^{\prime}\right), \lambda(f)(z)\left(x^{\prime \prime}\right)\right)=b\left(f\left(\left\langle z, x^{\prime}\right\rangle\right), f\left(\left\langle z, x^{\prime \prime}\right\rangle\right)\right)$. This proves that $\left\langle\lambda(f), \lambda_{0}(\varphi)\right\rangle(z) \in$ $\mathbf{L L}_{\mathrm{Met}}(X, Y)$.

Finally, any $z$ is contained in an open ball such that, whenever $z^{\prime}, z^{\prime \prime}$ belong to it, $\lambda_{1}(\varphi)\left(\left\langle z, c\left(z^{\prime}, z^{\prime \prime}\right)\right\rangle\right)(x) \geq$ $b\left(\lambda(f)\left(z^{\prime}\right)(x), \lambda(f)\left(z^{\prime \prime}\right)(x)\right)=b\left(f\left(\left\langle z^{\prime}, x\right\rangle\right), f\left(\left\langle z^{\prime \prime}, x\right\rangle\right)\right)$, so we can conclude that $\lambda(f, \varphi) \in \mathbf{L L}_{\mathrm{pMet}}\left(Z, \mathbf{L L}_{\mathrm{pMet}}(X, Y)\right)$. $(\Leftarrow)$ the map $\operatorname{ev}(\langle g, \psi\rangle, \chi)=\langle\operatorname{ev}(g), \operatorname{ev}(\psi, \chi)\rangle$ is defined by

$$
\begin{aligned}
\operatorname{ev}(f)(\langle z, x\rangle) & =f(z)(x) \\
\operatorname{ev}(\psi, \chi)(\langle z, x\rangle,\langle\zeta, \epsilon\rangle) & =\chi(\langle z, \zeta\rangle)(x)+\psi(z)(\langle x, \epsilon\rangle)
\end{aligned}
$$

The map ev $(\psi, \chi)$ is additive in its second variable. In fact we have

$$
\begin{aligned}
\mathrm{ev}(\psi, \chi)(\langle z, x\rangle,\langle 0,0\rangle) & =\chi(\langle z, 0\rangle)(x)+\psi(z)(\langle x, 0\rangle) \\
& =0+0=0
\end{aligned}
$$


and

$$
\begin{aligned}
& \operatorname{ev}(\psi, \chi)\left(\langle z, x\rangle,\left\langle\zeta+\zeta^{\prime}, \epsilon+\epsilon^{\prime}\right\rangle\right) \\
& =\chi\left(\left\langle z, \zeta+\zeta^{\prime}\right\rangle\right)(x)+\psi(z)\left(\left\langle x, \epsilon+\epsilon^{\prime}\right\rangle\right) \\
& =\chi(\langle z, \zeta\rangle)(x)+\chi\left(\left\langle z, \zeta^{\prime}\right)(x)+\psi(z)(\langle x, \epsilon\rangle)+\psi(z)\left(\left\langle x, \epsilon^{\prime}\right\rangle\right)\right. \\
& =\chi(\langle z, \zeta\rangle)(x)+\psi(z)(\langle x, \epsilon\rangle)+\chi\left(\left\langle z, \zeta^{\prime}\right)(x)+\psi(z)\left(\left\langle x, \epsilon^{\prime}\right\rangle\right)\right. \\
& =\operatorname{ev}(\psi, \chi)(\langle z, x\rangle,\langle\zeta, \epsilon\rangle)+\operatorname{ev}(\psi, \chi)\left(\langle z, x\rangle,\left\langle\zeta^{\prime}, \epsilon^{\prime}\right\rangle\right)
\end{aligned}
$$

Moreover, for all $z \in Z$ and $x \in X$ there exists $\zeta_{z} \gg 0, \epsilon_{x} \gg 0$ (which implies $\left\langle\zeta_{z}, \epsilon_{x}\right\rangle \gg 0$ ) such that whenever $c\left(z, z^{\prime}\right), c\left(z, z^{\prime \prime}\right) \leq \zeta_{z}$ and $a\left(x, x^{\prime}\right), a\left(x, x^{\prime \prime}\right) \leq \epsilon_{x}$

$$
\begin{aligned}
& \operatorname{ev}(\psi, \chi)(\langle z, x\rangle,\langle\zeta, \epsilon\rangle) \\
& \geq b\left(f\left(z^{\prime}\right)\left(x^{\prime}\right), f\left(z^{\prime}\right)\left(x^{\prime \prime}\right)\right)+b\left(f\left(z^{\prime}\right)\left(x^{\prime \prime}\right), f\left(z^{\prime \prime}\right)\left(x^{\prime \prime}\right)\right) \\
& \geq b\left(f\left(z^{\prime}\right)\left(x^{\prime}\right), f\left(z^{\prime \prime}\right)\left(x^{\prime \prime}\right)\right. \\
& =b\left(\operatorname{ev}(f)\left(\left\langle z^{\prime}, x^{\prime}\right\rangle\right), \operatorname{ev}(f)\left(\left\langle z^{\prime \prime}, x^{\prime \prime}\right\rangle\right)\right)
\end{aligned}
$$

We can thus conclude that ev $(\langle g, \psi\rangle, \chi) \in \mathbf{L L}_{\mathrm{pMet}}(Z \times X, Y)$.

It remains to show that $\lambda$ and ev inverse each-other:

- on one side we have $\left.\operatorname{ev}\left(\left\langle\lambda(f), \lambda_{0}(\varphi)\right\rangle, \lambda_{1}(\varphi)\right\rangle\right)=\left\langle\operatorname{ev}(\lambda(f)), \operatorname{ev}\left(\lambda_{0}(\varphi), \lambda_{1}(\varphi)\right)\right\rangle=\langle f, \varphi\rangle$, since $\mathrm{ev}\left(\lambda_{0}(\varphi), \lambda_{1}(\varphi)\right)(\langle z, x\rangle,\langle\zeta, \epsilon\rangle)=\varphi(\langle z, x\rangle,\langle\zeta, 0\rangle)+\varphi(\langle z, x\rangle,\langle 0, \epsilon\rangle)=\varphi(\langle z, x\rangle,\langle\zeta, \epsilon\rangle)$ by the additivity of $\varphi$.

- on the other side we have $\lambda(\operatorname{ev}(\langle g, \psi\rangle, \chi))=\lambda(\operatorname{ev}(g), \operatorname{ev}(\psi, \chi))=\left(\left\langle\lambda(\operatorname{ev}(g)), \lambda_{0}(\operatorname{ev}(\psi, \chi))\right\rangle, \lambda_{1}(\operatorname{ev}(\psi, \chi))\right\rangle=(\langle g, \psi\rangle, \chi)$, since $\lambda_{0}(\operatorname{ev}(\psi, \chi))(z)(\langle x, \epsilon\rangle)=\psi(z)(\langle x, \epsilon\rangle)+\chi(\langle z, 0\rangle)(x)=\psi(z)(\langle x, \epsilon\rangle)$ and $\lambda_{1}(\operatorname{ev}(\psi, \chi))(\langle z, \zeta\rangle)(x)=\psi(z)(\langle x, 0\rangle)+$ $\chi(\langle z, \zeta\rangle)(x)=\chi(\langle z, \zeta\rangle)(x)$.

The cartesian closure of $\mathbf{L L}_{\text {Met }}$ is proved as follows: if $f \in \mathbf{L L}_{\mathrm{Met}}(Z \times X, Y)$, then $f$ admits a family of LL-constants $\varphi$. Then for all $z \in Z, \lambda_{0}(\varphi)(z)$ is a family of LL-constants for $\lambda(f)(z)$, which implies that $\lambda(f)(z) \in \mathbf{L} \mathbf{L}_{\text {Met }}(X, Y)$; moreover, $\lambda_{1}(\varphi)$ is a family of LL-constants for the application $z \mapsto \lambda(f)(z)$, so we can conclude that $\lambda(f) \in \mathbf{L L}_{\mathrm{Met}}\left(Z, Y^{X}\right)$.

If now $f \in \mathbf{L L}_{\mathrm{Met}}\left(Z, Y^{X}\right)$, then for all $z \in Z$, the set of families of LL-constants for $f(z)$ is non-empty; by the axiom of choice, there exists then a function $\psi$ yielding, for all $z \in Z$, a family of LL-constants for $f(z)$. Moreover $f$ itself admits a family of LL-constants $\chi$. Then the map ev $(\psi, \chi)$ is a family of LL-constants for ev $(f)$, so we deduce $\operatorname{ev}(f) \in \mathbf{L L}_{\mathrm{Met}}(X, Y)$.

It remains to prove that $U$ is a cartesian closed functor. This descends from the following facts:

- $X \times Y / a \times b \simeq(X / a) \times(Y / b):$ in fact $\langle x, y\rangle \simeq_{a \times b}\left\langle x^{\prime}, y^{\prime}\right\rangle$ iff $x \simeq_{a} x^{\prime}$ and $y \simeq_{b} y^{\prime}$.

- $\mathbf{L L}_{\mathrm{pMet}}(X, Y) / b^{X} \simeq(Y / b)^{(X / a)}$ : first, observe that $(f, \varphi) \simeq_{b^{X}}(g, \psi)$ iff for all $x \in X, f(x) \simeq_{b} g(x)$ iff for all $x, y \in X$, $a(x, y)=0$ implies $f(x) \simeq_{b} g(y)$ (since $f, g$ are stable under $\simeq_{a}$-classes). Now, for all $\simeq_{a}$-stable functions $f, g$, let $f \sim g$ iff for all $x, y \in X, a(x, y)=0$ implies $f(x) \simeq_{b} g(y)$. Then the claim follows from the observation that the equivalence classes of $\sim$ are in bijection with the functions from $\simeq_{a}$-classes to $\simeq_{b}$-classes.

Finally, since for all pseudo-metric space $(X, Q, a)$ we have that $U a([x],[y])=a(x, y)$, from $b(f(y), f(z)) \leq \varphi(x, a(y, z))$ we deduce $U b(U f([y]), U f([z])) \leq \tilde{\varphi}([x], U a([y],[z]))$. We conclude then that $\tilde{\varphi}$ is a family of LL-constants for $U f$. 